2020-01

Power extraction by a water turbine in inviscid free surface flow with vertical shear

\author{
Lin, J
}

http://hdl.handle.net/10026.1/17671

10.1016/j.euromechflu.2019.10.002

European Journal of Mechanics - B/Fluids

Elsevier BV

All content in PEARL is protected by copyright law. Author manuscripts are made available in accordance with publisher policies. Please cite only the published version using the details provided on the item record or document. In the absence of an open licence (e.g. Creative Commons), permissions for further reuse of content should be sought from the publisher or author. 


\section{Journal Pre-proof}

Power extraction by a water turbine in inviscid free surface flow with vertical shear

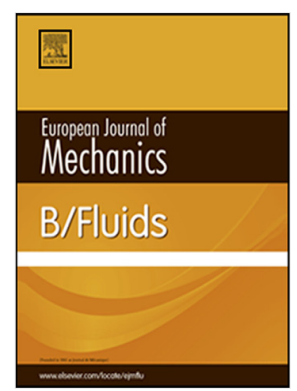

PII:

S0997-7546(19)30110-4

DOI: $\quad$ https://doi.org/10.1016/j.euromechflu.2019.10.002

Reference: EJMFLU 103554

To appear in: European Journal of Mechanics / B Fluids

Received date: 27 February 2019

Revised date : 20 August 2019

Accepted date : 3 October 2019

Please cite this article as: J. Lin, A.G.L. Borthwick, B. Lin et al., Power extraction by a water turbine in inviscid free surface flow with vertical shear, European Journal of Mechanics / B Fluids (2019), doi: https://doi.org/10.1016/j.euromechflu.2019.10.002.

This is a PDF file of an article that has undergone enhancements after acceptance, such as the addition of a cover page and metadata, and formatting for readability, but it is not yet the definitive version of record. This version will undergo additional copyediting, typesetting and review before it is published in its final form, but we are providing this version to give early visibility of the article. Please note that, during the production process, errors may be discovered which could affect the content, and all legal disclaimers that apply to the journal pertain.

(C) 2019 Published by Elsevier Masson SAS. 


\title{
Power extraction by a water turbine in inviscid free surface flow with vertical shear
}

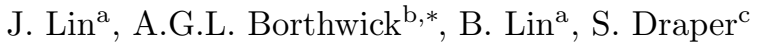 \\ ${ }^{a}$ State Key Laboratory of Hydroscience and Engineering, Tsinghua University, Beijing, China \\ ${ }^{b}$ Institute for Infrastructure and Environment, School of Engineering, The University of Edinburgh, The King's Buildings, \\ Edinburgh EH9 3JL, UK \\ ${ }^{c}$ School of Civil, Environmental and Mining Engineering, University of Western Australia, WA 6009, Australia
}

\begin{abstract}
Hydro-kinetic, tidal stream, and ocean current energy turbines operate in flows subject to vertical shear, which has an influence on the turbines, especially ones located near the bed. The gravity applied on a fluid is proportional to its density, thus the static pressure induced by gravity is enhanced by the higher density of water than air. Turbines are expected to be placed in fast moving, shallow flows. Hence the Froude number may be relatively high and changes to the free surface are likely, leading to additional flow confinement. In order to investigate the combined effect of vertical shear and gravity on idealized turbines, an extension of linear momentum actuator disc theory (LMADT) is used to estimate the thrust and power extracted by an idealized turbine for two types of free surface inviscid flow. It is assumed that there is fast pressure recovery and that the core flow contains self-similar velocity profiles. Results from a parameter study in which the velocity profiles and turbine settings are varied show that idealized turbines operate at higher efficiency under the effect of gravity, but operate at either higher or lower efficiency under shear flow. The proposed model can also be used to investigate energy extracted by turbines in a periodically spaced array, enabling better evaluation of array efficiency.
\end{abstract}

Keywords: Tidal stream energy, Three-dimensional LMADT, Shear flow, Free surface flow

\section{Introduction}

\subsection{Preamble}

Renewable energy devices designed to exploit the kinetic energy of moving water are invariably situated in free surface flows that are sheared in the vertical by the presence of bed and free surface boundary layers. 5 Flow shear is particularly important for turbines located near the bed. In the simple case of boundary layer flow over a flat bed, the horizontal stream-wise velocity component has a vertical structure that may be

\footnotetext{
*Corresponding author

Email address: Alistair.Borthwick@ed.ac.uk (A.G.L. Borthwick)
} 
approximated by a power or logarithmic law, with the velocity gradient being greatest close to the bed. Thus the efficiency of each single turbine in an array is influenced by the effect of shear in the incoming flow, and this should be important for the optimized spatial arrangement of the turbine array. Moreover, existing theories used for tidal turbines are mainly built on those developed for wind turbines, and so miss some of the unique features of water: its higher density, the importance of the free surface, etc. The density of water is three orders of magnitude larger than that of air, and the influence of gravity is thus more significant. Its effect in water becomes even larger when the water surface deformation is also significant, such as at a high blockage ratio or a large value of Froude number. To date, however, the overall effects of free surface shear flow on hydro-kinetic, tidal stream, and ocean current energy extraction have not been investigated thoroughly.

\subsection{LMADT Chronology}

Linear momentum actuator disc theory (LMADT) is one of the most popular methods used to describe energy addition or extraction from a flow, with applications ranging from the propulsion of ship propellers to the power of tidal turbines. The underlying theory dates back to Rankine (1865), who proposed use of a porous actuator disc to represent a ship's screw propeller (Okulov and van Kuik, 2012). In the mid to late 1800s many researchers attempted to analyse propeller thrust using actuator disc models, but it was not until 1889 that a correct description of such a flow was provided by Froude (1889) who included accelerations both upstream and downstream of the propeller in the analysis. This did not end the debate however, with different schools of thought remaining on how to model thrust on a propeller as an actuator disc using continuity, momentum, and energy principles.

In the early $20^{\text {th }} \mathrm{C}$, researchers also began to apply actuator disc theory to determine the power extracted by wind turbines. Lanchester (1915), Betz (1920), and Joukowsky (1920) adopted the basic principle of an actuator disc to estimate the power coefficient of a wind turbine, with the latter two researchers determining the well-known limiting ratio of $16 / 27$ in unbounded flow. Despite progress made in early wind turbine performance studies, the predictive capability of the classical actuator disc model turned out to be limited by assumptions of uniform oncoming flow and no lateral boundaries (Bahaj et al., 2012). Unfortunately, such ideal cases hardly exist in the real world for either wind or water stream turbines. In practice, alternative methods such as laboratory and field tests and numerical simulation provide further understanding (Draper et al., 2016). However, theoretical models based on LMADT retain their value because of their speed of execution and the fact that they provide the user with more comprehensive insight into generic problems of energy extraction by turbines. With this in mind, Wimshurst and Willden (2016) state that LMADT models provide a useful baseline for the more complicated field scenarios that need to be considered in practice.

When LMADT was first applied to the assessment of tidal stream energy, Garrett and Cummins (2007) introduced a volume-constrained actuator disc model (GC07 model) in order to deal with the blockage 
effect by treating the free surface as a rigid-lid. Their results highlighted the significance of tidal channel geometry on the performance of a tidal fence. By combining the GC07 model with an earlier 1D tidal channel model (Garrett and Cummins, 2005), Vennell (2010, 2011) determined optimal turbine settings and maximum efficiencies for turbine farms which fully blocked a channel. Nishino and Willden (2012, 2013) studied energy extraction from partially blocked channels by extending the volume-constrained model using the idea of scale decomposition, which is based on (mass, momentum, energy) conservation relationships at single turbine and array scales. All the foregoing models were limited to low Froude number cases because of the rigid-lid assumption. Meanwhile, a free surface flow actuator disc model without mixing was developed by Whelan et al. (2007, 2009), and this was later extended by Houlsby et al. (2008) who included the effect of downstream mixing in the analysis. Houlsby et al.'s model enabled the investigation of tidal stream energy extraction under different Froude number conditions, and permitted investigation of equally spaced tidal turbines (Draper, 2011). The aforementioned models assume uniform incoming flow, thus requiring the turbines to be located sufficiently far from lateral boundaries (i.e. top, bottom and side boundaries) and upstream turbines, which is not always the case in practice. Recently, Draper and Nishino (2014) developed an actuator disc model that considers the interaction of two rows of turbines by dividing the incoming flow into a uniform core section and a uniform bypass section. This model breaks the distance limit required for full mixing, and makes it possible to investigate densely spaced turbines. The model has been used to study optimal arrangements for single and two row arrays, as well as the influence of turbine arrangements on optimal spacing distances. Further progress in actuator disc theory has been achieved by Draper et al. (2016) who developed an inviscid shear model for freely-expanded and volume-constrained scenarios. This model provides a first glimpse into the effects of sheared velocity distributions on turbine efficiency, in rigidlid flows. The extension enables momentum theory to interpret approximately force variation and energy capture across a turbine for the first time.

Besides its direct application to tidal stream energy analysis, actuator disc theory is also useful for laboratory experiments and numerical simulations. For example, Barnsley and Wellicome (1990) presented a blockagecorrection methodology based on LMADT, versions of which are becoming widely used in tank experiments and numerical simulations (Bahaj et al., 2007; Gauthier et al., 2016; Kinsey and Dumas, 2017). The momentum actuator disc concept has also become a popular way of parametrizing turbines in numerical models, both in idealized cases (Hunter et al., 2015; Nash et al., 2015), and in real cases (Adcock et al., 2013; Karsten et al., 2013; Fallon et al., 2014; Nash et al., 2014; González-Gorbeña et al., 2015). Besides applications to water stream energy extraction, actuator disc theories have also been adopted to study hydrodynamic loads on submerged objects, such as currents acting on offshore structures (Taylor, 1991; Taylor et al., 2013; Santo et al., 2014). 


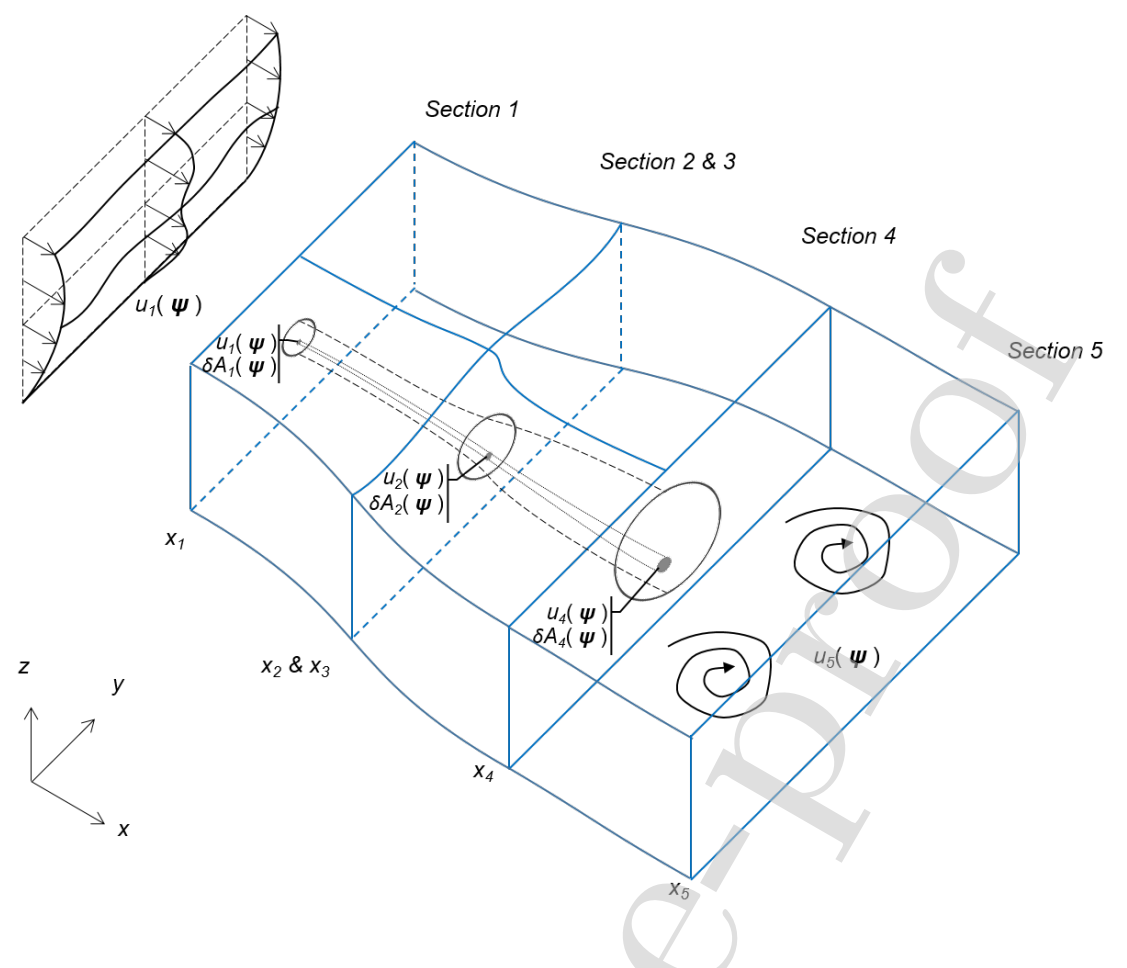

Figure 1: Overall view of non-uniform LMADT model

\subsection{Synopsis}

\section{Analytical model}

We first consider an actuator disc operating in an arbitrary shear flow, in water of finite depth. An overall control volume is fitted to the water body, whose lateral boundaries are defined by streamlines (Figure 1). The upstream boundary (marked as $x_{1}$ ) and downstream internal boundary (marked as $x_{4}$ ) are sufficiently 
far from the disc that the pressures are almost undisturbed.

We now focus on an infinitesimal stream tube, denoted by a parameter vector $\boldsymbol{\psi}$, which indicates both the location and discharge of the stream tube (i.e. $y=y(\boldsymbol{\psi}), z=z(\boldsymbol{\psi})$ and $d \boldsymbol{\psi}=u(\boldsymbol{\psi}) d A(\boldsymbol{\psi})$ at an arbitrary cross-section, where $A$ is the cross-sectional area). This is analogous to the stream function in two-dimensional and axisymmetric three-dimensional flows. For stream tubes intersecting with the actuator disc, there is a resistance $\delta T$ on the fluid. This force causes the flow to decelerate and thus the velocities at Section 2 and Section 4 are $u_{2}(\boldsymbol{\psi})=u\left(x_{2}, \boldsymbol{\psi}\right)=\alpha_{2}(\boldsymbol{\psi}) u_{1}(\boldsymbol{\psi})$ and $u_{4}(\boldsymbol{\psi})=u\left(x_{4}, \boldsymbol{\psi}\right)=\alpha_{4}(\boldsymbol{\psi}) u_{1}(\boldsymbol{\psi})$ respectively, where $\alpha_{2}(\boldsymbol{\psi}), \alpha_{4}(\boldsymbol{\psi}) \in[0,1]$, and $u_{1}(\boldsymbol{\psi})$ is the velocity of the same steam tube at Section 1 . On the other hand, the fluid in stream tubes bypassing the disc accelerates and its velocity changes from $u_{2}(\boldsymbol{\psi})=u\left(x_{2}, \boldsymbol{\psi}\right)=\beta_{2}(\boldsymbol{\psi}) u_{1}(\boldsymbol{\psi})$ to $u_{4}(\boldsymbol{\psi})=u\left(x_{4}, \boldsymbol{\psi}\right)=\beta_{4}(\boldsymbol{\psi}) u_{1}(\boldsymbol{\psi})$ as it passes through the two crosssections, noting that $\beta_{2}(\boldsymbol{\psi}), \beta_{4}(\boldsymbol{\psi})>1$. The adoption of $\boldsymbol{\psi}$ can be interpreted as a projection from Cartesian to stream function coordinates, as shown in figure 2. An important point to be noted here is the concrete form of the forementioned parameter vector (or stream function) $\boldsymbol{\psi}$. Based on knowledge from vector calculus, an incompressible flow field can be defined as the curl of a vector field (noted as $\boldsymbol{\psi}$ )

$$
\boldsymbol{u}=\nabla \times \boldsymbol{\psi}
$$

Without loss of generality, Masatsuka (2013) write the vector field $\boldsymbol{\psi}$ in the form of $\boldsymbol{\psi}=\psi \operatorname{grad} \chi($ where $\psi$ and $\chi$ are both scalar fields) and transform Equation 1 into

$$
\boldsymbol{u}=\operatorname{grad} \psi \times \operatorname{grad} \chi
$$

which means the velocity field $\boldsymbol{u}$ is tangent to the two families of surfaces defined by $\psi$ and $\chi$, and its magnitude $u=|\operatorname{grad} \psi||\operatorname{grad} \psi| \sin \theta$, where $\theta$ indicates the angle between normals of the two surfaces. For a cross-section perpendicular to the velocity field (as with the Sections shown in Figure 1), the area (on the cross-section) bounded by two $\psi$-surfaces and two $\chi$-surfaces can be calculated, wirtten as

$$
d A=\frac{d \psi d \chi}{|\operatorname{grad} \psi||\operatorname{grad} \psi| \sin \theta}
$$

and thus the discharge in the stream tube (represented using $d \boldsymbol{\psi}$ ) bounded by the four surfaces is

$$
d \boldsymbol{\psi}=d Q=u(\boldsymbol{\psi}) d A(\boldsymbol{\psi})=d \psi d \chi
$$

where $\mathrm{Q}$ is discharge. At this point, we find a parameter vector $\boldsymbol{\psi}=(\psi, \chi)$ indicating both the location and discharge of the stream tube, as required at the beginning of this section $(\S 2)$. 

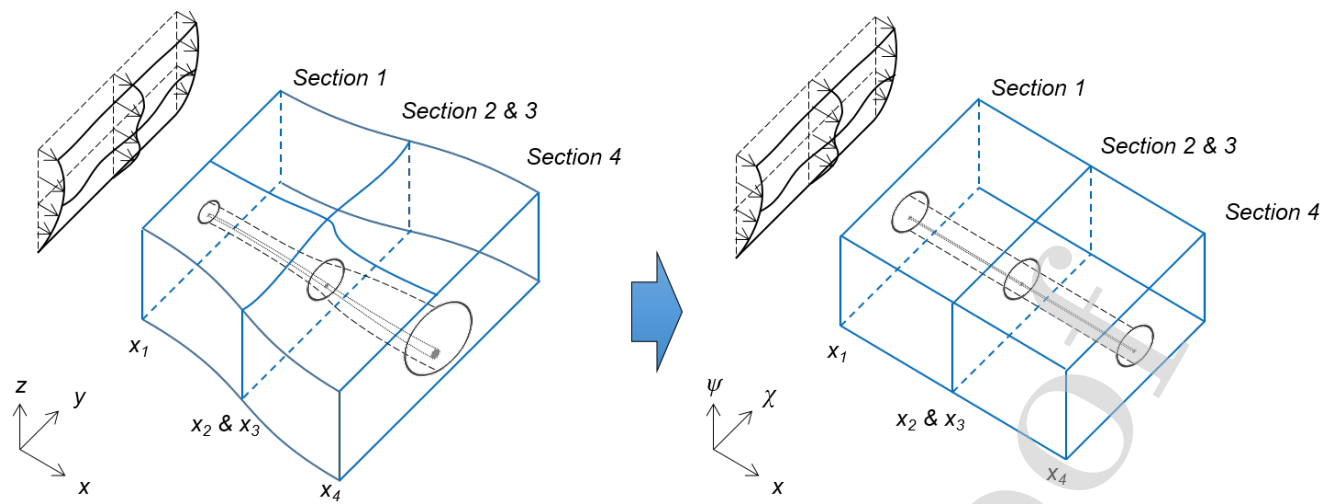

Figure 2: Projection from Cartesian to stream function coordinates (left: $x-y-z$ system; right: $x-\psi-\chi$ system). Blue solid lines demarcate control-volume boundaries separated by streamlines, Section 1 and Section 4, while black dashed lines indicate lateral boundaries of the core flow and bypass flow. The transformation enables formulation of conservation laws within an infinitesimal stream tube, see $\S 2.1-\S 2.3$.

\section{1. $3 D$ model}

In order to analyse the proposed flow field in Figure 1, the first assumption (A1) required is that the wake pressure recovers quickly such that piezometric heads are uniform at Section 1 and Section 4 . This assumption implies that the flow is hydrostatic at Section 1 and Section 4 , and the water surface is uniform in the transverse direction. Hence,

$$
p_{1}(\psi)+\rho g z_{1}(\psi)=p_{1}+\rho g z_{1}
$$

and

$$
p_{4}(\boldsymbol{\psi})+\rho g z_{4}(\boldsymbol{\psi})=p_{4}+\rho g z_{4}
$$

where $p_{x}(\boldsymbol{\psi})$ is the pressure at the intersection of Section $x$ and Streamline $\boldsymbol{\psi}, z_{x}(\boldsymbol{\psi})$ is the vertical coordinate of the intersection, and $\psi$ is the analogous stream function which defines the specified streamline.

(1) Mass conservation

A tidal period is of the scale of days (e.g. 12.5 hours), which is much longer than the periods of tidal turbine rotors. For this reason, when analyzing the force applied on a rotor, the flow is often taken to be approximately steady. So tidal turbines are designed to operate in quasi-steady flows. For mass conservation to be satisfied, the discharge must remain constant within any steam tube bounded by streamlines. Specifically, for the core flow at Sections 1, 2 and 4, we have

$$
\mathrm{d} \boldsymbol{\psi}_{t_{1}}=\mathrm{d} \boldsymbol{\psi}_{t_{2}}=\mathrm{d} \boldsymbol{\psi}_{t_{4}}=\mathrm{d} \boldsymbol{\psi}_{t}
$$


and for the bypass flow at Sections 1, 2, and 4, we have

$$
\mathrm{d} \boldsymbol{\psi}_{b_{1}}=\mathrm{d} \boldsymbol{\psi}_{b_{2}}=\mathrm{d} \boldsymbol{\psi}_{b_{4}}=\mathrm{d} \boldsymbol{\psi}_{b}
$$

and

(2) Energy conservation

$$
\alpha_{2}(\boldsymbol{\psi}) u_{1}(\boldsymbol{\psi}) \frac{\mathrm{d} \boldsymbol{\psi}}{u_{2}(\boldsymbol{\psi})}-\alpha_{4}(\boldsymbol{\psi}) u_{1}(\boldsymbol{\psi}) \frac{\mathrm{d} \boldsymbol{\psi}}{u_{4}(\boldsymbol{\psi})}=0, \quad \boldsymbol{\psi} \in\left\{\boldsymbol{\psi}_{t}\right\}
$$

and

$$
u_{1}(\boldsymbol{\psi}) \frac{\mathrm{d} \boldsymbol{\psi}}{u_{1}(\boldsymbol{\psi})}-\beta_{4}(\boldsymbol{\psi}) u_{1}(\boldsymbol{\psi}) \frac{\mathrm{d} \boldsymbol{\psi}}{u_{4}(\boldsymbol{\psi})}=0, \quad \boldsymbol{\psi} \in\left\{\boldsymbol{\psi}_{b}\right\}
$$

where $\left\{\boldsymbol{\psi}_{t}\right\}$ and $\left\{\boldsymbol{\psi}_{b}\right\}$ represent the sets composed of the core flow streamlines and bypass flow streamlines respectively. Eliminating $u_{1}(\boldsymbol{\psi})$ from Equations 9 and 10, and integrating give

$$
\iint_{t} \alpha_{2}(\boldsymbol{\psi}) \mathrm{d} A_{2}-\iint_{t} \alpha_{4}(\boldsymbol{\psi}) \mathrm{d} A_{4}=0
$$

Here, mixing occurs only after Section 4 and no shear effect exists within the control volume between Section 1 and Section 4. These prerequisites ensure that the actuator disc is the sole source of energy variation between Section 1 and Section 4. Thus, the energy of the core flow is conserved between Section 1 and Section 2, and Section 3 and Section 4. Hence,

$$
p_{1}(\boldsymbol{\psi})+\rho g z_{1}(\boldsymbol{\psi})+\frac{\rho\left[u_{1}(\boldsymbol{\psi})\right]^{2}}{2}=p_{2}(\boldsymbol{\psi})+\rho g z_{2}(\boldsymbol{\psi})+\frac{\rho\left[u_{2}(\boldsymbol{\psi})\right]^{2}}{2}, \quad \boldsymbol{\psi} \in\left\{\boldsymbol{\psi}_{t}\right\}
$$

and

$$
p_{3}(\boldsymbol{\psi})+\rho g z_{3}(\boldsymbol{\psi})+\frac{\rho\left[u_{3}(\boldsymbol{\psi})\right]^{2}}{2}=p_{4}(\boldsymbol{\psi})+\rho g z_{4}(\boldsymbol{\psi})+\frac{\rho\left[u_{4}(\boldsymbol{\psi})\right]^{2}}{2}, \quad \boldsymbol{\psi} \in\left\{\boldsymbol{\psi}_{t}\right\} .
$$

For the bypass flow, energy is conserved between Section 1 and Section 4, and so

$$
p_{1}(\boldsymbol{\psi})+\rho g z_{1}(\boldsymbol{\psi})+\frac{\rho\left[u_{1}(\boldsymbol{\psi})\right]^{2}}{2}=p_{4}(\boldsymbol{\psi})+\rho g z_{4}(\boldsymbol{\psi})+\frac{\rho\left[u_{4}(\boldsymbol{\psi})\right]^{2}}{2}, \quad \boldsymbol{\psi} \in\left\{\boldsymbol{\psi}_{b}\right\}
$$

The pressure difference across the disc can then be obtained by combining Equations 13-15,

$$
\delta T=p_{2}(\boldsymbol{\psi})-p_{3}(\boldsymbol{\psi})=\Delta p^{\prime}+\frac{\rho}{2}\left[u_{1}(\boldsymbol{\psi})\right]^{2}\left(1-\left[\alpha_{4}(\boldsymbol{\psi})\right]^{2}\right), \quad \boldsymbol{\psi} \in\left\{\boldsymbol{\psi}_{t}\right\}
$$

$$
\Delta p^{\prime}=\frac{\rho}{2}\left[u_{1}(\boldsymbol{\psi})\right]^{2}\left(\left[\beta_{4}(\boldsymbol{\psi})\right]^{2}-1\right), \quad \boldsymbol{\psi} \in\left\{\boldsymbol{\psi}_{b}\right\}
$$


The piezometric head difference is uniform over the cross-section according to assumption A1. Thus $\Delta p^{\prime}$ can be obtained from $u_{1}(\boldsymbol{\psi})$ and $\beta_{4}(\boldsymbol{\psi})$ along any stream tube in the bypass flow. As described by Draper et al. (2016), a single parameter representation of $\beta_{4}(\boldsymbol{\psi})$ can be obtained by manipulating Equation 17 to give

$$
\beta_{4}(\boldsymbol{\psi})=\left[1+\frac{u_{1}^{\prime 2}}{\left[u_{1}(\boldsymbol{\psi})\right]^{2}}\left(\beta_{4}^{\prime 2}-1\right)\right]^{1 / 2}, \quad \boldsymbol{\psi} \in\left\{\boldsymbol{\psi}_{b}\right\},
$$
between Section 1 and Section 4 (including both the core flow and the bypass flow) leads to

$$
X-T=\iint_{t \& b} \rho\left[u_{4}(\boldsymbol{\psi})\right]^{2} \frac{\mathrm{d} \boldsymbol{\psi}}{u_{4}(\boldsymbol{\psi})}-\iint_{t \& b} \rho\left[u_{1}(\boldsymbol{\psi})^{2}\right] \frac{\mathrm{d} \boldsymbol{\psi}}{u_{1}(\boldsymbol{\psi})}
$$

where

$$
\begin{aligned}
X & =\oiint_{C V} p_{x} \mathrm{~d} s \\
& =\iint_{C V_{\text {end }}} p_{x}(\boldsymbol{\psi}) \frac{\mathrm{d} \boldsymbol{\psi}}{u_{i}(\boldsymbol{\psi})}+\iint_{C V_{\text {side }}} p_{x} \mathrm{~d} s \\
& =\iint_{t \& b} p_{1}(\boldsymbol{\psi}) \frac{\mathrm{d} \boldsymbol{\psi}}{u_{1}(\boldsymbol{\psi})}-\iint_{t \& b} p_{4}(\boldsymbol{\psi}) \frac{\mathrm{d} \boldsymbol{\psi}}{u_{4}(\boldsymbol{\psi})}+\iint_{C V_{\text {side }}} p_{x} \mathrm{~d} s
\end{aligned}
$$

in which $p_{x}$ is the $x$ pressure component at the surfaces of the overall control volume, $C V_{\text {end }}$ represents the two end surfaces of the control volume and $C V_{\text {side }}$ represents the lateral surfaces of the control volume. Equation 20 can be simplified by ignoring certain small terms, as discussed by Sørensen (2011). In the present study, the pressure force ( $x$ component) is neglected on lateral surfaces of the overall control body, which comprise a water-air interface, a flat bed, and two parallel side boundaries. At the air water-air interface, the pressure force can be neglected because of the huge density difference between water and air. At the flat bed, the $x$ component of the pressure force is equal to zero because the flat bed is parallel to the $x$ axis. At the side wall boundaries, the inclusion of bypass flow in the overall control body partly compensates for streamline deformation. It is reasonable to neglect the $x$-component of the pressure on the two side boundaries when the lateral boundaries are located far from the disc or when the actuator discs are periodically spaced, which is usually the case in the present study. This allows us to ignore the last term in Equation 20. Eliminating $T$ and $X$ in Equation 19 using Equation 16 and 20 gives

$$
\begin{aligned}
& \iint_{t \& b} p_{1}(\boldsymbol{\psi}) \frac{\mathrm{d} \boldsymbol{\psi}}{u_{1}(\boldsymbol{\psi})}-\iint_{t \& b} p_{4}(\boldsymbol{\psi}) \frac{\mathrm{d} \boldsymbol{\psi}}{u_{4}(\boldsymbol{\psi})}-\iint_{t}\left(\Delta p^{\prime}+\frac{\rho}{2}\left[u_{1}(\boldsymbol{\psi})\right]^{2}\left(1-\left[\alpha_{4}(\boldsymbol{\psi})\right]^{2}\right)\right) \frac{\mathrm{d} \boldsymbol{\psi}}{u_{2}(\boldsymbol{\psi})} \\
& =\iint_{t \& b} \rho\left[u_{4}(\boldsymbol{\psi})\right]^{2} \frac{\mathrm{d} \boldsymbol{\psi}}{u_{4}(\boldsymbol{\psi})}-\iint_{t \& b} \rho\left[u_{1}(\boldsymbol{\psi})^{2}\right] \frac{\mathrm{d} \boldsymbol{\psi}}{u_{1}(\boldsymbol{\psi})} .
\end{aligned}
$$


After manipulation, 21 becomes

$$
\begin{aligned}
& \iint_{t \& b} p_{1}(\boldsymbol{\psi}) \frac{\mathrm{d} \boldsymbol{\psi}}{u_{1}(\boldsymbol{\psi})}-\iint_{t \& b} p_{4}(\boldsymbol{\psi}) \frac{\mathrm{d} \boldsymbol{\psi}}{u_{4}(\boldsymbol{\psi})}-\iint_{t}\left(\Delta p^{\prime}+\frac{\rho}{2}\left[u_{1}(\boldsymbol{\psi})\right]^{2}\left(1-\left[\alpha_{4}(\boldsymbol{\psi})\right]^{2}\right)\right) \frac{\mathrm{d} \boldsymbol{\psi}}{u_{2}(\boldsymbol{\psi})} \\
& =\rho \iint_{t}\left[u_{1}(\boldsymbol{\psi})\right]\left(\left[\alpha_{4}(\boldsymbol{\psi})\right]-1\right) \mathrm{d} \boldsymbol{\psi}+\rho \iint_{b}\left[u_{1}(\boldsymbol{\psi})\right]\left(\left[\beta_{4}(\boldsymbol{\psi})\right]-1\right) \mathrm{d} \boldsymbol{\psi}
\end{aligned}
$$

where the first and second terms represent total pressures at Section 1 and Section 4 respectively, the third term represents the pressure difference across the disc, the fourth and last terms are the net $x$-component momentum changes of the core flow and bypass flow. Furthermore, the first two terms can be substituted by a simplified relationship between the pressure and cross-sectional area. If the lateral boundaries of the overall control volume are assumed to be vertical at Section 1 and Section 4 (a reasonable assumption when the lateral boundaries are far from the disc or the actuator discs are periodically spaced), then the pressure terms can be written

$$
\iint_{t \& b} p_{i}(\boldsymbol{\psi}) \frac{\mathrm{d} \boldsymbol{\psi}}{u_{i}(\boldsymbol{\psi})}=\frac{\rho g A_{i}^{2}}{2 w_{i}}
$$

at Section $i(i=1,4)$, and are equal to half the product of the piezometric head and the cross-sectional area because of the hydrostatic assumption at the two cross-sections. Then the following relationship between $A_{1}$ and $A_{4}$ can be obtained using 17 and 23 , giving

$$
A_{4}=\frac{w_{4}}{w_{1}} A_{1}-\frac{w_{4}}{2 g} u_{1}^{\prime 2}\left(\beta_{4}^{\prime 2}-1\right)
$$

where $w_{i}(i=1,4)$ is the width of the cross-section at $i$, and $A_{1}$ and $A_{4}$ are the cross-sectional areas at Section 1 and Section 4 respectively. Equations 23 and 24 enable the pressure terms to be represented using $A_{1}$, which is a known parameter under specified conditions.

In the final step of the derivation, Equations 17, 23, and 24 are substituted into Equation 22 and manipulated to give

$$
\begin{aligned}
& \frac{g A_{1}^{2}}{2 w_{1}}-\frac{g}{2 w_{4}}\left(\frac{w_{4}}{w_{1}} A_{1}-\frac{w_{4}}{2 g} u_{1}^{\prime 2}\left(\beta_{4}^{\prime 2}-1\right)\right)^{2}-\frac{\Delta p^{\prime}}{\rho} B A_{1}-\frac{1}{2} \iint_{t} u_{1}(\boldsymbol{\psi}) \frac{1-\left[\alpha_{4}(\boldsymbol{\psi})\right]^{2}}{\alpha_{2}(\boldsymbol{\psi})} \mathrm{d} \boldsymbol{\psi} \\
= & \iint_{t} u_{1}(\boldsymbol{\psi})\left(\alpha_{4}(\boldsymbol{\psi})-1\right) \mathrm{d} \boldsymbol{\psi}+\iint_{b} u_{1}(\boldsymbol{\psi})\left(\left[1+\frac{u_{1}^{\prime 2}}{\left[u_{1}(\boldsymbol{\psi})\right]^{2}}\left(\beta_{4}^{\prime 2}-1\right)\right]^{1 / 2}-1\right) \mathrm{d} \boldsymbol{\psi}
\end{aligned}
$$

where $B$ is the blockage ratio, defined as the ratio between the actuator disc area $A_{d i s c}$ and the upstream cross-sectional area $A_{1}$ of the overall control volume. Meanwhile, Equations 11, 12, 18, and 24 can be combined to give the following implicit constraint for $a_{4}(\psi)$

$$
\iint_{t} \frac{1}{\alpha_{4}(\boldsymbol{\psi})} \frac{\mathrm{d} \boldsymbol{\psi}}{u_{1}(\boldsymbol{\psi})}+\iint_{b}\left[1+\frac{u_{1}^{\prime 2}}{\left[u_{1}(\boldsymbol{\psi})\right]^{2}}\left(\beta_{4}^{\prime 2}-1\right)\right]^{-1 / 2} \frac{\mathrm{d} \boldsymbol{\psi}}{u_{1}(\boldsymbol{\psi})}=\frac{w_{4}}{w_{1}} A_{1}-\frac{w_{4}}{2 g} u_{1}^{\prime 2}\left(\beta_{4}^{\prime 2}-1\right) .
$$


For a given case, the cross-sectional area $A_{1}$, and section widths $w_{1}$ and $w_{4}$ can be determined once the control volume is prescribed. The remaining variables are $\beta_{4}^{\prime}, \alpha_{2}(\boldsymbol{\psi})$ and $a_{4}(\boldsymbol{\psi})$. If the distribution of $\alpha_{4}(\boldsymbol{\psi})$ can be obtained (i.e. $\alpha_{4}(\boldsymbol{\psi})=\alpha_{4}^{\prime} f(\boldsymbol{\psi})$ and $f(\boldsymbol{\psi})$ is known, where $\alpha_{4}^{\prime}$ is a constant and $f(\boldsymbol{\psi})$ is the distribution function), then $\alpha_{4}^{\prime}$ can be represented with $\beta_{4}^{\prime}$ using Equation 26. Next, an explicit relationship between $\alpha_{4}(\boldsymbol{\psi})$ and $\beta_{4}^{\prime}$ can be obtained. Thus the model comprising Equations 25 and 26 can be simplified to a univariant system in $\beta_{4}^{\prime}$, involving case-specific parameters indicating the velocity deficit distribution $\alpha_{2}(\boldsymbol{\psi})$.

\subsection{5 D model}

As a preliminary study, the model is further simplified by assuming the core wake profiles to be selfsimilar (assumption A2). This assumption restricts the analysis to scenarios where $\alpha_{2}$ and $\alpha_{4}$ are uniform, similar to cases of resistance where geometric blockage and shear are not excessive (following Draper et al. $(2016))$, so that

$$
\alpha_{2}(\psi)=\alpha_{2}
$$

and

$$
\alpha_{4}(\psi)=\alpha_{4}
$$

Adopting assumption A2, Equation 25 can be simplified to give

$$
\begin{aligned}
& \frac{g A_{1}^{2}}{2 w_{1}}-\frac{g}{2 w_{4}}\left(\frac{w_{4}}{w_{1}} A_{1}-\frac{w_{4}}{2 g} u_{1}^{\prime 2}\left(\beta_{4}^{\prime 2}-1\right)\right)^{2}-\frac{\Delta p^{\prime}}{\rho} B A_{1}-\frac{1-\alpha_{4}^{2}}{2 \alpha_{2}} \iint_{t} u_{1}(\boldsymbol{\psi}) \mathrm{d} \boldsymbol{\psi} \\
= & \left(\alpha_{4}-1\right) \iint_{t} u_{1}(\boldsymbol{\psi}) \mathrm{d} \boldsymbol{\psi}+\iint_{b} u_{1}(\boldsymbol{\psi})\left(\left[1+\frac{u_{1}^{\prime 2}}{\left[u_{1}(\boldsymbol{\psi})\right]^{2}}\left(\beta_{4}^{\prime 2}-1\right)\right]^{1 / 2}-1\right) \mathrm{d} \boldsymbol{\psi} .
\end{aligned}
$$

Meanwhile, Equations 11, 12, 18, and 24 can be combined to give the following explicit relationship between $a_{2}$ and $a_{4}$

$$
\frac{\alpha_{2}}{\alpha_{4}} B A_{1}+\iint_{b}\left[1+\frac{u_{1}^{\prime 2}}{\left[u_{1}(\boldsymbol{\psi})\right]^{2}}\left(\beta_{4}^{\prime 2}-1\right)\right]^{-1 / 2} \frac{\mathrm{d} \boldsymbol{\psi}}{u_{1}(\boldsymbol{\psi})}=\frac{w_{4}}{w_{1}} A_{1}-\frac{w_{4}}{2 g} u_{1}^{\prime 2}\left(\beta_{4}^{\prime 2}-1\right) .
$$

By eliminating either $\alpha_{4}$ (or $\alpha_{2}$ ) in Equation 29 using Equation 30, the model can be simplified to a single variable $\left(\beta_{4}^{\prime}\right)$ equation, involving case-specific parameters comprising the velocity deficit coefficient $\alpha_{2}$ (or $\alpha_{4}$ ), blockage ratio $B$ (or disc area), and an upstream velocity profile.

When investigating the thrust and power performance of a water turbine in shear flow, it is useful to distinguish between different definitions of average $n$-th power upstream velocities, i.e.

$$
\overline{U_{*}^{n}}=\frac{1}{\alpha_{2} A_{d i s c}} \iint_{t}\left[u_{1}(\boldsymbol{\psi})\right]^{n} \frac{\mathrm{d} \boldsymbol{\psi}}{u_{1}(\boldsymbol{\psi})}
$$

and

$$
\overline{U^{n}}=\frac{1}{A_{d i s c}} \iint_{t^{\prime}}\left[u_{1}(z)\right]^{n} \mathrm{~d} A
$$

where $A_{\text {disc }}$ is the area of the actuator disc, $t$ represents the upstream flow passing through the strip, and $t^{\prime}$ the upstream flow within the area range of the strip. The two definitions are equivalent (both equal to $\bar{U}^{n}$ ) 
when the flow is uniform. However, differences exist when shear occurs, causing the upstream flow location and velocity magnitude to vary. According to the two definitions, the thrust and power coefficients can be defined respectively as

$$
C_{t}=\frac{T}{\frac{1}{2} \rho A_{d i s c} \overline{U_{*}^{2}}}, C_{p}=\frac{P}{\frac{1}{2} \rho A_{d i s c} \overline{U_{*}^{3}}}
$$

and

$$
C_{t}^{\prime}=\frac{T}{\frac{1}{2} \rho A_{d i s c} \overline{U^{2}}}, C_{p}^{\prime}=\frac{P}{\frac{1}{2} \rho A_{\text {disc }} \overline{U^{3}}}
$$

Manipulating Equation 36 gives

$$
\frac{g}{2 w_{5}} A_{5}^{3}+\left(-\frac{g A_{1}^{2}}{2 w_{1}}+\frac{T}{\rho}-\iint_{t \& b} u_{1}(\boldsymbol{\psi}) \mathrm{d} \boldsymbol{\psi}\right) A_{5}+\iint_{t \& b} \gamma_{5} u_{1}(\boldsymbol{\psi}) \mathrm{d} \boldsymbol{\psi}=0 .
$$

This is a cubic equation in cross-sectional area $A_{5}$, which can be solved once the thrust $T$ is determined from the shear LMADT model. Thus the total power removed from water per width can be represented (using $A_{5}$ ) as

$$
P_{T o t}=\rho g\left(A 1 / w 1-A 5 / w_{5}\right) \iint_{t \& b} \mathrm{~d} \boldsymbol{\psi}-\left(\left(A_{1} / A_{5}\right)^{2}-1\right) \iint_{t \& b} \frac{\rho u_{1}(\boldsymbol{\psi})^{2}}{2} \mathrm{~d} \boldsymbol{\psi} .
$$

The basin efficiency can be calculated using

$$
\eta=\frac{P}{P_{T o t}}=P /\left(\rho g\left(A 1 / w 1-A 5 / w_{5}\right) \iint_{t \& b} \mathrm{~d} \boldsymbol{\psi}-\left(\left(A_{1} / A_{5}\right)^{2}-1\right) \iint_{t \& b} \frac{\rho u_{1}(\boldsymbol{\psi})^{2}}{2} \mathrm{~d} \boldsymbol{\psi}\right) .
$$


To derive the 2.5 D model, the core wake profiles are presumed self-similar. Before further simplification and application of the model, it is valuable to have a brief discussion of the validity of this assumption. We introduce a local resistance coefficient $k(\boldsymbol{\psi})$, such that the thrust applied on a micro stream tube can be written,

$$
\delta T=\frac{1}{2} \rho k(\boldsymbol{\psi})\left[\alpha_{2}(\boldsymbol{\psi}) u_{1}(\boldsymbol{\psi})\right]^{2}, \quad \boldsymbol{\psi} \in\left\{\boldsymbol{\psi}_{t}\right\} .
$$

Comparing Equation 41 with Equations 16, 17 and 18, the distribution of $k$ can be written,

$$
\left.k(\boldsymbol{\psi})=\frac{1}{\left[\alpha_{2}(\boldsymbol{\psi})\right]^{2}}\left(1-\left[\alpha_{4}(\boldsymbol{\psi})\right]^{2}+\frac{u_{1}^{\prime 2}}{\left[u_{1}(\boldsymbol{\psi})\right]^{2}}\left(\beta_{4}^{\prime 2}-1\right)\right)\right), \quad \boldsymbol{\psi} \in\left\{\boldsymbol{\psi}_{t}\right\} .
$$

It can be seen that the resistance needed to form a target core wake profile $\alpha_{2}(\boldsymbol{\psi})$ or $\alpha_{4}(\boldsymbol{\psi})$ is determined by both the upstream velocity profile $u_{1}(\boldsymbol{\psi})$ and accleration of bypass flow (described by $u_{1}^{\prime}$ and $\beta_{4}^{\prime}$ ). As with the discussion by Draper et al. (2016) for two dimensional shear flow cases, when self-similar core wakes are required, $k(\boldsymbol{\psi})$ reduces to the following function of $u_{1}(\boldsymbol{\psi})$

$$
\left.k(\boldsymbol{\psi})=\frac{1}{\alpha_{2}^{2}}\left(1-\alpha_{4}^{2}+\frac{u_{1}^{\prime 2}}{\left[u_{1}(\boldsymbol{\psi})\right]^{2}}\left(\beta_{4}^{\prime 2}-1\right)\right)\right), \quad \boldsymbol{\psi} \in\left\{\boldsymbol{\psi}_{t}\right\} .
$$

Equation 43 defines the discs for which the presented model is valid. Such discs may differ from more comdiscussed in $\S 4$. 


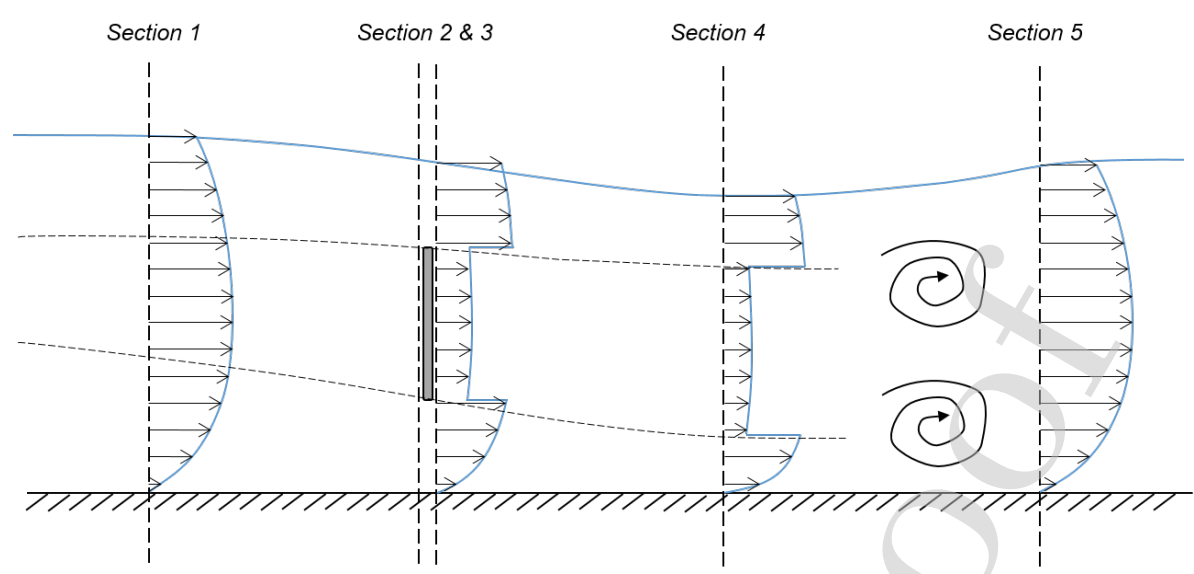

Figure 3: Two-dimensional LMADT model in shear flow

\subsection{2 $D$ model}

For cases where the actuator disc (strip) is very wide (in the transverse direction) and vertical velocity profiles are similar along the strip (such as a tidal fence), the flow presents quasi-two-dimensional features (as shown in Figure 3). Thus, the model can be further reduced to two dimensions. Equation 29 is then rewritten

$$
\begin{aligned}
& \frac{1}{2} g h_{1}^{2}-\frac{1}{2} g\left(h_{1}-\frac{1}{2 g} u_{1}^{\prime 2}\left(\beta_{4}^{\prime 2}-1\right)\right)^{2}-\frac{1}{2} u_{1}^{\prime 2}\left(\beta_{4}^{\prime 2}-1\right) B h_{1}-\frac{1-\alpha_{4}^{2}}{2 \alpha_{2}} \int_{t} u_{1}(\psi) \mathrm{d} \psi \\
= & \left(\alpha_{4}-1\right) \int_{t} u_{1}(\psi) \mathrm{d} \psi+\int_{b} u_{1}(\psi)\left(\left[1+\frac{u_{1}^{\prime 2}}{\left[u_{1}(\psi)\right]^{2}}\left(\beta_{4}^{\prime 2}-1\right)\right]^{1 / 2}-1\right) \mathrm{d} \psi
\end{aligned}
$$

where $h_{1}$ is the water depth at Section 1 and $\psi$ is two-dimensional stream function. Equation 30 becomes

$$
\frac{\alpha_{2}}{\alpha_{4}} B h_{1}+\int_{b}\left[1+\frac{u_{1}^{\prime 2}}{\left[u_{1}(\psi)\right]^{2}}\left(\beta_{4}^{\prime 2}-1\right)\right]^{-1 / 2} \frac{\mathrm{d} \psi}{u_{1}(\psi)}=h_{1}-\frac{1}{2 g} u_{1}^{\prime 2}\left(\beta_{4}^{\prime 2}-1\right) .
$$

This simplifies to give

$$
\begin{aligned}
& \frac{1}{2} g h_{1}^{2}-\frac{1}{2} g\left(h_{1}-\frac{1}{2 g} u_{1}^{\prime 2}\left(\beta_{4}^{\prime 2}-1\right)\right)^{2}-\frac{1}{2} u_{1}^{\prime 2}\left(\beta_{4}^{\prime 2}-1\right) B h_{1}-\frac{1-\alpha_{4}^{2}}{2 \alpha_{2}} I_{1} \\
= & \left(\alpha_{4}-1\right) I_{1}+I_{2}
\end{aligned}
$$

255 and

$$
\frac{\alpha_{2}}{\alpha_{4}} B h_{1}+I_{0}=h_{1}-\frac{1}{2 g} u_{1}^{\prime 2}\left(\beta_{4}^{\prime 2}-1\right),
$$

where

$$
\begin{gathered}
I_{0}=\int_{b}\left[1+\frac{u_{1}^{\prime 2}}{\left[u_{1}(\psi)\right]^{2}}\left(\beta_{4}^{\prime 2}-1\right)\right]^{-1 / 2} \frac{\mathrm{d} \psi}{u_{1}(\psi)}, \\
I_{1}=\int_{t} u_{1}(\psi) \mathrm{d} \psi
\end{gathered}
$$


and

$$
I_{2}=\int_{b} u_{1}(\psi)\left(\left[1+\frac{u_{1}^{\prime 2}}{\left[u_{1}(\psi)\right]^{2}}\left(\beta_{4}^{\prime 2}-1\right)\right]^{1 / 2}-1\right) \mathrm{d} \psi .
$$

Again, a univariant equation of $\beta_{4}^{\prime}$ can be obtained by eliminating $\alpha_{4}$ (or $\alpha_{2}$ ) in Equation 46 using Equation

47. A bisection method is adopted by the authors to solve the equation. Similar to the three-dimensional scenario, definitions of average $n$-th power upstream velocities are written as

$$
\overline{U_{*}^{n}}=\frac{1}{\alpha_{2} l} \int_{t}\left[u_{1}(\psi)\right]^{n} \frac{\mathrm{d} \psi}{u_{1}(\psi)}
$$

and

$$
\overline{U^{n}}=\frac{1}{l} \int_{t^{\prime}}\left[u_{1}(z)\right]^{n} \mathrm{~d} z
$$

where $l$ is the height of the strip. Two-dimensional thrust and power coefficients can be defined respectively as

$$
C_{t}=\frac{T}{\frac{1}{2} \rho l \overline{U_{*}^{2}}}, C_{p}=\frac{P}{\frac{1}{2} \rho l \overline{U_{*}^{3}}}
$$

and

$$
C_{t}^{\prime}=\frac{T}{\frac{1}{2} \rho l \overline{U^{2}}}, C_{p}^{\prime}=\frac{P}{\frac{1}{2} \rho l \overline{U^{3}}}
$$

where $T=\int_{t} \delta T \frac{\mathrm{d} \psi}{\alpha_{2} u_{1}(\psi)}$ is the thrust applied on the strip per unit length, and $P=\int_{t} \delta T \alpha_{2} u_{1}(\psi) \frac{\mathrm{d} \psi}{\alpha_{2} u_{1}(\psi)}$ is the power extracted by the strip per unit length. Considering energy loss due to wake mixing in a similar fashion to the three-dimensional case, the velocity profile at Section 5 in the two-dimensional case can be written as

$$
u_{5}(\psi)=\gamma_{5} u_{1}(\psi)
$$

Applying momentum conservation between the two sections results in

$$
\frac{1}{2} g h_{1}^{2}-\frac{1}{2} g\left(h_{1}-\Delta h\right)^{2}-\frac{T}{\rho}=\int_{t \& b} \gamma_{5} u_{1}(\psi) \mathrm{d} \psi-\int_{t \& b} u_{1}(\psi) \mathrm{d} \psi,
$$

where $\Delta h$ is the water level drop between Section 1 and Section 5 , and $T$ is the thrust per unit width. Meanwhile, mass conservation between the two sections requires

$$
\gamma_{5}=\frac{h_{1}}{h_{1}-\Delta h} .
$$

Substituting Equation 57 into Equation 56 and manipulating gives

$$
\frac{1}{2} \frac{\Delta h^{3}}{h_{1}^{3}}-\frac{3}{2} \frac{\Delta h^{2}}{h_{1}^{2}}+\left(1-F r^{2}+\frac{T}{\rho g h_{1}^{2}}\right) \frac{\Delta h}{h_{1}}-\frac{T}{\rho g h_{1}^{2}}=0,
$$

where $F r^{\prime}=\sqrt{\frac{\int_{t \& b} u_{1}(\psi) \mathrm{d} \psi}{g h_{1}} / h_{1}}$. This is a cubic equation in water level difference $\Delta h$ which can be solved once the thrust $T$ is given by the shear LMADT model. The total power removed from water per width can be represented (using $\Delta h$ ) as

$$
P_{T o t}=\rho g \Delta h \int_{t \& b} \mathrm{~d} \psi-\left(\left(\frac{h_{1}}{h_{1}-\Delta h}\right)^{2}-1\right) \int_{t \& b} \frac{\rho u_{1}(\psi)^{2}}{2} \mathrm{~d} \psi
$$


and the basin efficiency given by

$$
\eta=\frac{P}{P_{\text {Tot }}}=\frac{P}{\rho g \Delta h \int_{t \& b} \mathrm{~d} \psi}\left(1-F r^{\prime \prime 2} \frac{1-(1 / 2) \Delta h / h_{1}}{\left(1-\Delta h / h_{1}\right)^{2}}\right)^{-1},
$$

where $F r^{\prime \prime}=\sqrt{\int_{t \& b} \frac{u_{1}(\psi)^{2}}{g h_{1}} \mathrm{~d} \psi / \int_{t \& b} \mathrm{~d} \psi}$.

\section{Demonstration cases}

and

$$
I_{2}=U^{2}\left(\beta_{4}-1\right)\left(\frac{1}{B}-\alpha_{2}\right) l,
$$

where $l$ is the height of the strip and $l / B=h_{1}$. Then Equations 46 and 47 can be written as

$$
\begin{aligned}
& \frac{1}{2} g\left[\left(\frac{l}{B}\right)^{2}-\left(\frac{l}{B}-\frac{1}{2 g} U^{2}\left(\beta_{4}^{2}-1\right)\right)^{2}\right]-\frac{1}{2} U^{2} l\left(\beta_{4}^{2}-\alpha_{4}^{2}\right) \\
= & U^{2} l \alpha_{2}\left(\alpha_{4}-1\right)+U^{2} l\left(\beta_{4}-1\right)\left(\frac{1}{B}-\alpha_{2}\right)
\end{aligned}
$$

and

$$
\frac{\alpha_{2}}{\alpha_{4}} l+\frac{\left(1 / B-\alpha_{2}\right)}{\beta_{4}} l=\frac{l}{B}-\frac{1}{2 g} U^{2}\left(\beta_{4}^{2}-1\right),
$$


which are equivalent to the volume-pressure constrained LMADT model proposed by Houlsby et al. (2008) and Whelan et al. $(2007,2009)$. If $\alpha_{2}$ (or alternatively $\alpha_{4}$ ) is selected to represent the flow resistance of the actuator strip, then the strip's thrust and power performance can be evaluated once the blockage ratio $B$ and Froude number $\mathrm{Fr}$ are specified. Figure 4 compares power coefficients obtained using the proposed model with those from Houlsby et al.'s volume-pressure constrained LMADT model. The two sets of results agree exactly, implying that the two models are equivalent for cases where the incoming flow is uniform.

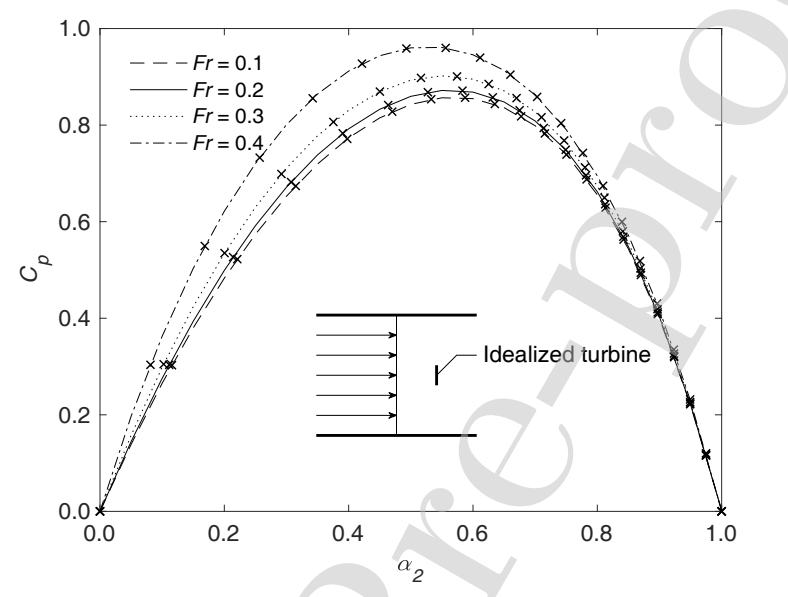

Figure 4: Comparisons between results from Houlsby et al.'s (2008), Whelan et al.'s (2007,2009) model (crosses) and the present model (lines) for $F r=0.1,0.2,0.3,0.4$, at blockage ratio $\hat{B}=1 / 6$

Figure 5 shows the blockage ratio $B$ and Froude number $F r$ parameter spaces over which physically admissible results can be obtained for specified values of $\alpha_{2}$. Each dashed line separates the figure into a lower left physically admissible region and upper right physically inadmissible region. An actuator operating at lower $\alpha_{2}$ results in a smaller admissible region (in terms of $B$ and $F r$ ). This can be explained by considering the limitation encountered by the free surface in balancing the strip. As $\alpha_{2}$ decreases, the strip has a stronger influence on the flow, which needs to be balanced by a larger background piezometric head gradient $\Delta p^{\prime}$ between the upstream and downstream ends of the strip. However, adjustment of the downstream flow regime alone cannot achieve this target at extremely small $\alpha_{2}$ values. This implies that the upstream flow must also be changed. Such scenarios can be compared to flows passing through a Venturi flume. A throat that is too narrow causes the upstream water level to rise until an equilibrium state is reached. It should be mentioned that the whole of the practically important solution space for tidal stream energy projects (blue dashed box, corresponding to $F r<0.2$ and $B<0.2)$ lies within the admissible regions. 


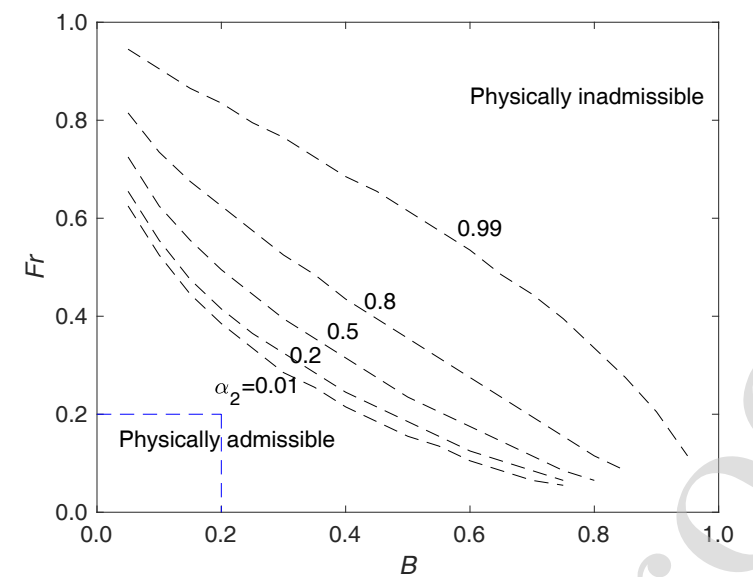

Figure 5: Boundaries of physically admissible Froude numbers and blockage ratios for $\alpha_{2}=0.01,0.2,0.5,0.8,0.99$ (the blue dashed box delineates the practically important space for tidal stream energy projects)

\subsection{D Power law shear flow}

Power law velocity profiles were originally used as empirical fits to data from pipe experiments conducted by Nikuradse and later used to model boundary layers on flat plates (Schlichting, 1955), for which both symmetric and asymmetric shear flows can be described. Selected extreme (linear \& uniform) and oftenused (1/5 \& 1/7) power law distributions will be adopted for demonstration purposes in the following subsections. Considering a symmetric shear flow, the power law approximation to the upstream velocity profile can be written as

$$
u_{1}(z)=U\left(1-2 \frac{|z| B}{l}\right)^{n}
$$

where $z$ is the vertical elevation taken as positive upwards from the centre of the strip (i.e. from mid-depth of the upstream flow). Thus the discharge $\psi$ between the bed and $\mathrm{z}$ can be obtained by integration as

$$
\psi(z)=\int_{0}^{z} u_{1}(z) \mathrm{d} z= \begin{cases}\frac{U l}{2 B(n+1)}\left(1+2 \frac{z B}{l}\right)^{n+1} & -\frac{l}{2 B} \leqslant z \leqslant 0 \\ \frac{U l}{2 B(n+1)}\left(1-2 \frac{z B}{l}\right)^{n+1} & 0 \leqslant z \leqslant \frac{l}{2 B}\end{cases}
$$

Representing $z$ in terms of $\psi$ using Equation 69 and substituting into Equation 68 gives

$$
u_{1}(\psi)= \begin{cases}U\left(\frac{2 B(n+1)}{U l}\left(\frac{\psi_{2}}{x}+\psi\right)\right)^{n /(n+1)} & -\frac{\psi_{2}}{2} \leqslant \psi \leqslant 0 \\ U\left(\frac{2-2 B(n+1)}{l} U^{1 / n}\left(\frac{\psi_{2}}{x}+\psi\right)\right)^{n /(n+1)} & 0 \leqslant \psi \leqslant \frac{\psi_{2}}{2},\end{cases}
$$

where

$$
\left\{\begin{array}{l}
\psi_{1}=\frac{U l}{B(n+1)}-\frac{U l}{B(n+1)}\left(1-a_{2} B\right)^{n+1} \\
\psi_{2}=\frac{U l}{B(n+1)}
\end{array}\right.
$$


in which $\psi_{1}$ defines the discharge passing through the strip and $\psi_{2}$ defines the total discharge through the control volume. Substitution of the above results into Equation 48 to 50 gives values for $I_{0}, I_{1}$ and $I_{2}$, then $\alpha_{4}$ (or $\alpha_{2}$ if $\alpha_{4}$ is specified to represent the flow resistance of the strip) can be calculated using Equation 47, and $\beta_{4}^{\prime}$ can be determined by solving Equation 46. These coefficients finally enable us to evaluate the performance of the strip using Equations 53 and 54 .

Figure 6 shows the dependence of power coefficient $C_{p}$ on $\alpha_{2}$ given by Equation 53 for symmetric power law profiles of different shapes $(n=0,1 / 7,1 / 5,1)$. For comparison against results from Draper et al. (2016), we set the geometric blockage ratio $B=1 / 6$ and $1 / 2$, and the Froude number $F r=0.1$ (defined using maximum velocity $U$ ). The dashed lines present the corresponding uniform flow results where $n=0$, obtained equally from either the general power law model (in the present subsection) or the uniform model (in the previous subsection). It can be seen from the figures that increasing shear (as $n$ increases), causes the power coefficient to decrease (in this case where the strips are located at mid-depth). The optimal power coefficients for $B=1 / 6$ and $1 / 2$ drop by $29 \%$ and $63 \%$ for the extreme shear profile $(n=1$, i.e. linear profile) with respect to the uniform flow profile, in a similar fashion to volume-constrained flows (Draper et al., 2016).
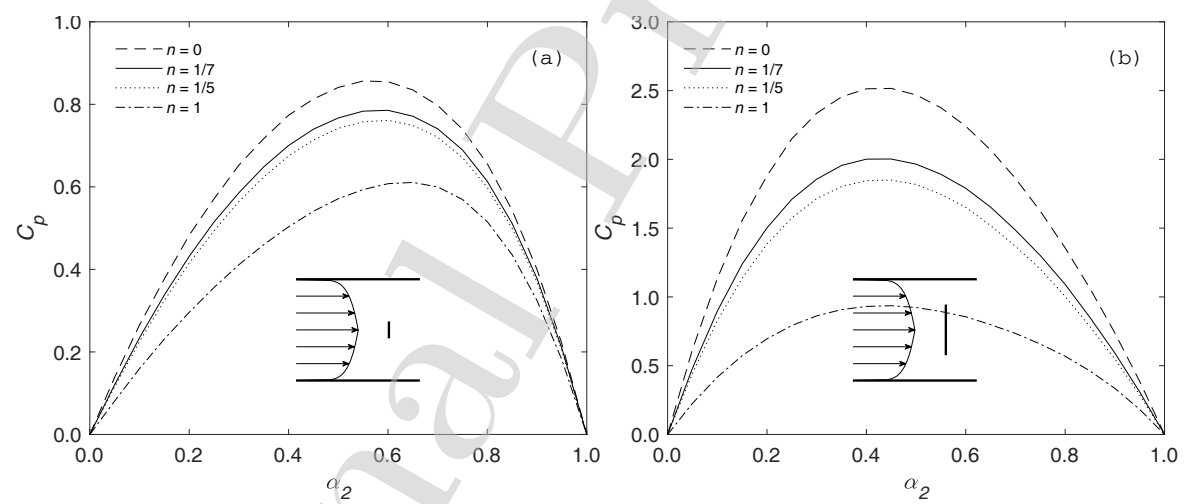

Figure 6: Variation in power coefficient $C_{p}$ with $\alpha_{2}$ for power law velocity profiles $(n=0,1 / 7,1 / 5,1)$, at $F r=0.1$ for: $(a)$ $B=1 / 6 ;$ and $(b) B=1 / 2$. The disc is deployed at water mid-depth, where inflow is vertically symmetrical

Unlike a volume-constrained flow, whose velocity scale has no influence on the power coefficient of the actuator for a specific profile shape, the velocity is important in a free surface flow. More exactly, the Froude number is a primary factor because it reflects the amount by which the free surface will deform. Figure 7 shows the relationship between the power coefficient $C_{p}$ and $\alpha_{2}$ for linear shear flows at Froude numbers ranging from 0.1 to 0.5 , and includes results from the volume-constrained shear LMADT model of Draper et al. (2016). Figure 7(a) shows that the values of power coefficient predicted by the two models converge when the strip influence is small (i.e. for large $\alpha_{2}$ and small $B$ ), especially for subcritical flows at low Froude 
numbers. However, gravity-induced improvements in power coefficient become significant as $B$ increases from $1 / 6$ to $1 / 2$. Furthermore, the predictions by the free surface model tend asymptotically to those by the volume-constrained model as $\mathrm{Fr} \rightarrow 0$. This phenomenon is related to the milder free surface changes that occur at lower Froude numbers. On the other hand, volume-constrained constant density flows can be thought of as occurring in a medium of infinitely large elastic modulus, where wave speeds are infinite, and so the ratio of water particle velocity to wave speed approaches zero, analogous to the situation as $\mathrm{Fr} \rightarrow 0$. Figures 6 and 7 also indicate that higher values of optimal power coefficient are obtained as the blockage ratio is increased from $1 / 6$ to $1 / 2$. However, a sufficiently large blockage ratio combined with strong influence of the disc on the flow (i.e. small $\alpha_{2}$ ) results in disappearance of physically admissible solutions to the model, as shown in Figure 7(b). This is because the upstream flow has to change in order to reach steady state, as discussed in Section 3.1.
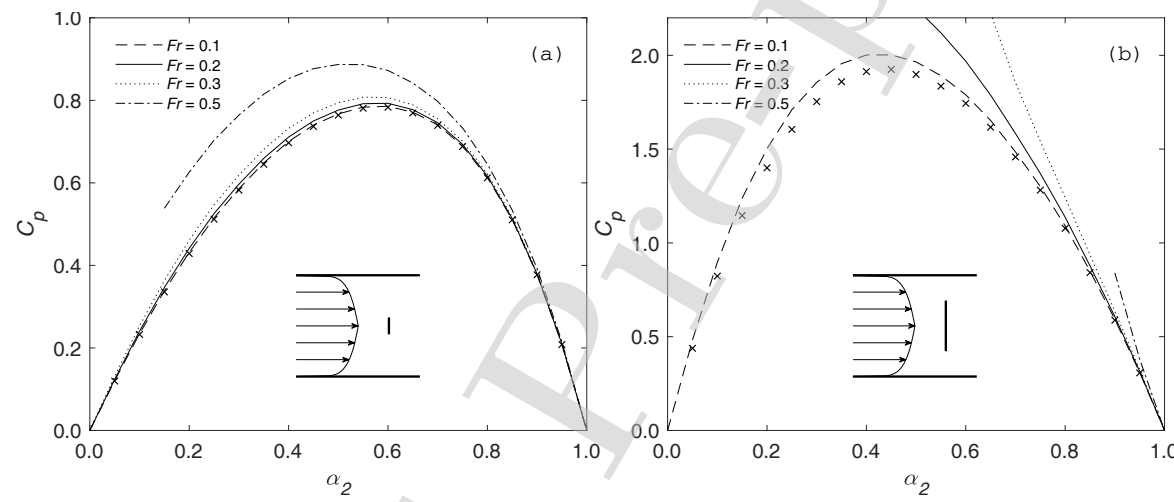

Figure 7: Variation in power coefficient $C_{p}$ with $\alpha_{2}$ for power law velocity profile $n=1 / 7$ at $F r=0.1,0.2,0.3,0.5$ for: (a) $B=1 / 6$; and $(b) B=1 / 2$. The crosses are results from volume-constrained LMADT model (Draper et al., 2016). The disc is deployed at water mid-depth, where inflow is vertically symmetrical

For boundary layer flows induced by bed friction with negligible free surface stresses, the asymmetric power law provides a category of simple, frequently-used distributions for velocity profiles (Bahaj et al., 2012), which can be written as

$$
u_{1}\left(z_{b}\right)=U\left(\frac{z_{b} B}{l}\right)^{n}
$$

where the elevation $z_{b}$ is 0 at the bed and taken positive upwards. Thus the discharge $\psi$ between the bed and a given level $z_{b}$ is given by

$$
\psi\left(z_{b}\right)=\int_{0}^{z_{b}} u_{1}\left(z_{b}\right) \mathrm{d} z_{b}=\frac{U l}{B(n+1)}\left(\frac{B z_{b}}{l}\right)^{n+1} .
$$

Representing $z_{b}$ in terms of $\psi$ using Equation 73 and substituting into Equation 72 gives

$$
u_{1}(\psi)=U^{1 /(n+1)}\left(\frac{B(n+1) \psi}{l}\right)^{n /(n+1)} .
$$


For asymmetric shear flows, we use a constant value of depth-averaged velocity $\bar{U}$ in the present parameter study. Using Equation 73 for a given depth-averaged velocity $\bar{U}$, the maximum velocity is expressed as

$$
U=(n+1) \bar{U}
$$

In a similar manner to the analysis of symmetric power law profiles, the functions $\psi_{1}^{\prime}, \psi_{2}^{\prime}$ and $\psi_{3}^{\prime}$ are used to represent discharges passing through different parts of the control volume, where $\psi_{2}^{\prime}-\psi_{1}^{\prime}$ defines the discharge passing through the strip, $\psi_{1}^{\prime}$ defines the lower bypass discharge and $\psi_{3}^{\prime}$ defines the total discharge. $\psi_{1}^{\prime}$, $\psi_{2}^{\prime}$ and $\psi_{3}^{\prime}$ are given by

$$
\left\{\begin{array}{l}
\psi_{1}^{\prime}=\frac{U l}{B(n+1)}\left(\frac{z_{d} B}{l}-\frac{a_{2} B}{2}\right)^{n+1} \\
\psi_{2}^{\prime}=\frac{U l}{B(n+1)}\left(\frac{z_{d} B}{l}+\frac{a_{2} B}{2}\right)^{n+1} \\
\psi_{3}^{\prime}=\frac{U l}{B(n+1)} .
\end{array}\right.
$$

Substitution of the above into Equations 48 to 50 enables calculation of $I_{0}, I_{1}$ and $I_{2}$, after which $\alpha_{4}$ and $\beta_{4}^{\prime}$ are obtained from Equation 47 and Equation 46. The performance of the strip is evaluated using Equations 53.

Figure 8 illustrates the dependence of power coefficient $C_{p}$ on coefficient $\alpha_{2}$ for strips located at middepth, with the velocity described by various asymmetric power law profiles $(n=0,1 / 7,1 / 5,1)$, at a geometric blockage ratio $B=1 / 6$. Here, the Froude number of free surface flow with an asymmetric velocity profile is defined using the depth-averaged velocity $\bar{U}$, and has a value $F r=0.1$. The dashed line presents the uniform flow $(n=0)$ results obtained for the same discharge. It can be seen that increasing flow shear (as the value of $n$ rises) causes the power coefficient to decrease at a similar scale to that obtained in symmetric power law shear flows. The optimal power coefficients obtained for $B=1 / 6$ are reduced by $6 \%, 9 \%, 23 \%$ for $1 / 7$ power law shear flow, $1 / 5$ power law shear flow and linear shear flow respectively .

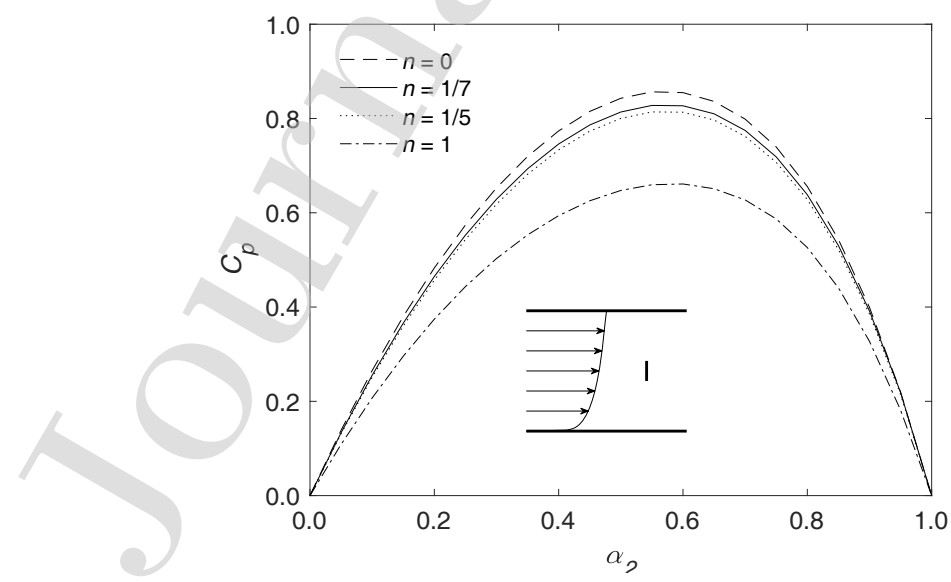

Figure 8: Variation in power coefficient $C_{p}$ with $\alpha_{2}$ for asymmetric power law velocity profiles $(n=0,1 / 7,1 / 5,1)$, at blockage ratio $B=1 / 6$ and Froude number $F r=0.1$. The disc is deployed at mid-depth of the power-law shear flow 
Figure 9 presents the power coefficient distributions with $\alpha_{2}$ for asymmetric power law shear flows where $n=1 / 7$, at Froude numbers $F r=0.1,0.2,0.3,0.5$. For a strip located at mid-depth, gravity effects raise the magnitude of power coefficient, especially for strips that have strong influence on the core flow velocity (i.e. small $\alpha_{2}$ ). As $\alpha_{2}$ decreases and $\mathrm{Fr}$ increases, more significant water level drops occur between Section 1 and Section 4. This pressure gradient acts as an additional source of extractable energy within the control volume.

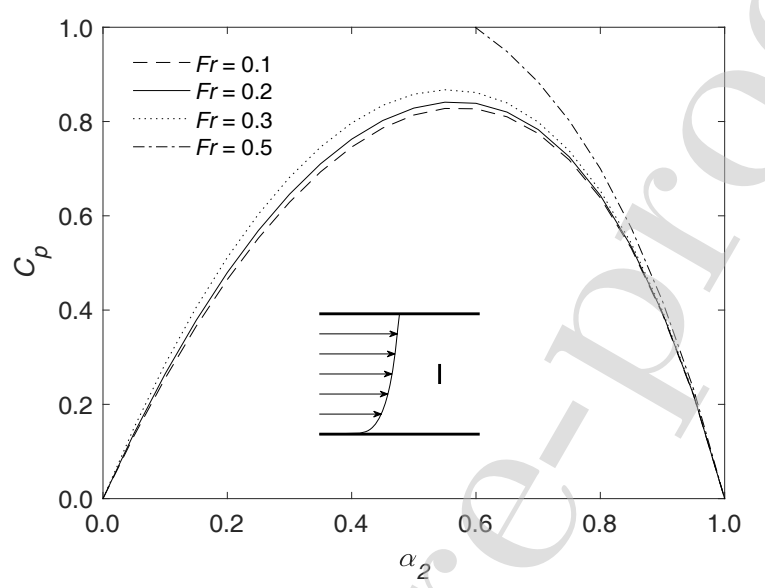

Figure 9: Variation in power coefficient $C_{p}$ with $\alpha_{2}$ for an asymmetric power law velocity profile with $n=1 / 7$, at blockage ratio $B=1 / 6$ and Froude numbers $F r=0.1,0.2,0.3,0.5$. The disc is deployed at mid-depth of the power-law shear flow
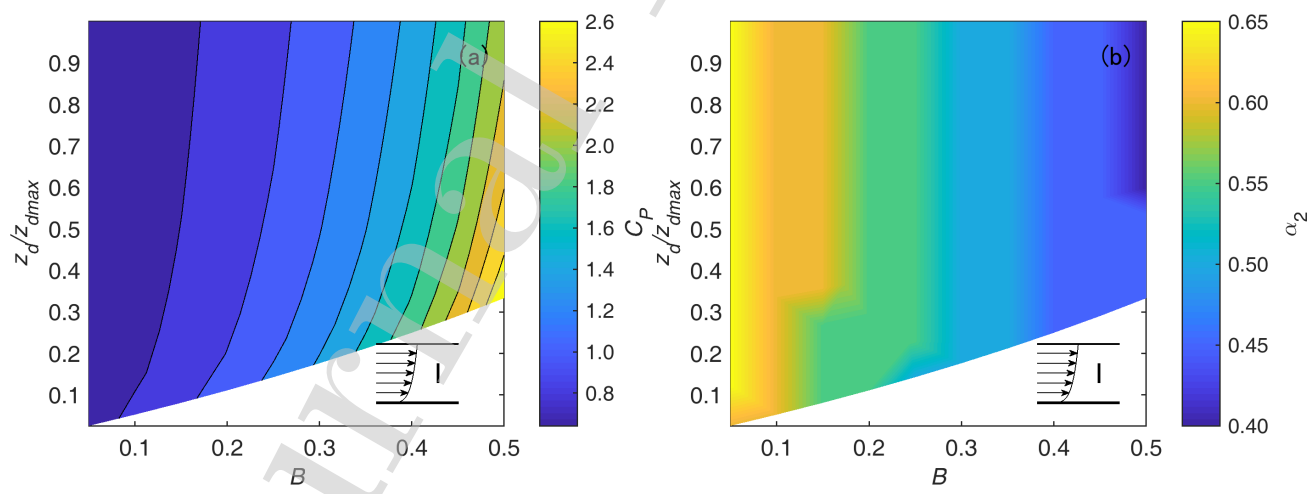

Figure 10: (a) Maximum power coefficient $C_{p}$ and (b) corresponding $\alpha_{2}$ for different blockage ratios $B$ and non-dimensional strip positions $z_{d} / z_{d m a x}$, at Froude number $F r=0.1$ and power law velocity profile $n=1 / 7$. The disc is deployed at different elevations in the power-law shear flow for varing blockage ratio

Figure 10 shows contours of maximum power coefficient $C_{p}$ and corresponding $\alpha_{2}$ with respect to blockage ratio $B$ and non-dimensional strip position $z_{d} / z_{d \max }$, at $F r=0.1$ and for an asymmetric power law velocity profile with $n=1 / 7$. Figure 10 (a) indicates that a higher peak power coefficient is achieved as the strip 
is moved downwards. This phenomenon can be attributed to the low velocity region near the bed, which increases the velocity ratio between bypass and core flows. Meanwhile, Figure 10(b) implies that weaker strips (i.e. higher $\alpha_{2}$ ) are required to achieve optimum energy extraction as the strip elevation increases, especially at low values of blockage ratio. It should be noted that blockage effects invariably improve strip performance until the upstream flow becomes unstable. Higher blockage ratios require stronger strips (smaller $\left.\alpha_{2}\right)$.

\subsection{D Logarithmic shear flow}

For logarithmic shear flows (Schlichting, 1955), the upstream velocity profile can be written as

$$
u_{1}\left(z_{b}\right)=\left\{\begin{array}{rr}
\frac{u_{*}}{\kappa} \ln \left(\frac{z_{b}}{z_{0}}\right), & z_{b} \geqslant z_{0} \\
0, & z_{b}<z_{0}
\end{array}\right.
$$

where $\kappa$ is the von Kármán constant, $u_{*}$ is friction velocity, and $z_{0}$ is bed roughness height. Integrating the velocity from the bed at $z=0$ to the strip centre location $z=z_{b}$, the discharge $\psi$ is expressed as

$$
\psi(z)=\int_{0}^{z_{b}} u_{1}\left(z_{b}\right) \mathrm{d} z_{b}=\left\{\begin{array}{r}
\frac{z_{b}\left(-1+\ln \left(z_{b} / z_{0}\right)\right) u_{*}}{\kappa}+\frac{z_{0} u_{*}}{\kappa}, \quad z_{b} \geqslant z_{0} \\
0, \quad z_{b}<z_{0} .
\end{array}\right.
$$

Representing $z_{b}$ in terms of $\psi$ using Equation 78 and substituting into Equation 77 gives

$$
u_{1}(\psi)=\left\{\begin{array}{rr}
\frac{u_{*}}{\kappa} \ln \left(\frac{\kappa \psi-z_{0} u_{*}}{z_{0} W\left(\frac{\kappa \psi-z_{0} u_{*}}{\mathrm{e} z_{0} u_{*}}\right) u_{*}}\right), & \psi>0 \\
0, & \psi=0
\end{array}\right.
$$

where $W(x)$ is the Lambert $W$ function, the inverse solution to $f(z)=z e^{z}$. For a specified depth-averaged velocity $\bar{U}$, the friction velocity $u_{*}$ can be determined (after manipulating Equation 78 ) from

$$
u_{*}=\frac{\kappa}{-1+\ln \left(h_{1} / z_{0}\right)+z_{0} / h_{1}} \bar{U}
$$

The discharge coefficients $\psi_{1}^{\prime}, \psi_{2}^{\prime}$ and $\psi_{3}^{\prime}$ are defined in the same way as for the asymmetric power law cases, using

$$
\left\{\begin{array}{l}
\psi_{1}^{\prime}=\frac{\left(z_{d}-a_{2} l / 2\right)\left(-1+\ln \left(\left(z_{d}-a_{2} l / 2\right) / z_{0}\right)\right) u_{*}}{\kappa}+\frac{z_{0} u_{*}}{\kappa} \\
\psi_{2}^{\prime}=\frac{\left(z_{d}+a_{2} l / 2\right)\left(-1+\ln \left(\left(z_{d}+a_{2} l / 2\right) / z_{0}\right)\right) u_{*}}{\kappa}+\frac{z_{0} u_{*}}{\kappa} \\
\psi_{3}^{\prime}=\frac{(l / B)\left(-1+\ln \left((l / B) / z_{0}\right)\right) u_{*}}{\kappa}+\frac{z_{0} u_{*}}{\kappa} .
\end{array}\right.
$$

Following the same calculation procedure, the above results can be substituted into Equations 48 to 50 to calculate $I_{0}, I_{1}$ and $I_{2}$, and $\alpha_{4}$ and $\beta_{4}^{\prime}$ can be solved in sequence using Equations 47 and Equation 46 . The strip performances are evaluated using Equations 53. 
For natural and engineering flows, the roughness height $z_{0}$ can be estimated on the basis of Nikuradse's experiments, with $z_{0}=k_{s} / 30$ providing a satisfactory fit to hydrodynamically rough flows, where $k_{s}$ can be calculated from $k_{s}=2.5 d_{50}$ and $d_{50}$ is the median size of the bed material (Soulsby, 1997). Following the categorization proposed by Wentworth (1922), we select $d_{50}=1 \mathrm{~mm}$ and $64 \mathrm{~mm}$ as representative diameters for sediment particles comprising sand and gravel beds. Figure 11 shows the variation of power coefficient $C_{p}$ with $\alpha_{2}$ for logarithmic shear flows over the sand and gravel beds at different Froude numbers $F r=0.1,0.2,0.3,0.5$. Here the strip is located at mid-depth. As expected, gravity effects again improve power performance at moderate values of $\alpha_{2}$ but are also prone to be unstable, as previously found in the power law cases. The power performances of the strips are similar for both classes of shear flows. This is because the two profiles are approximations to actual velocity profiles, and the curves are similar to each other.
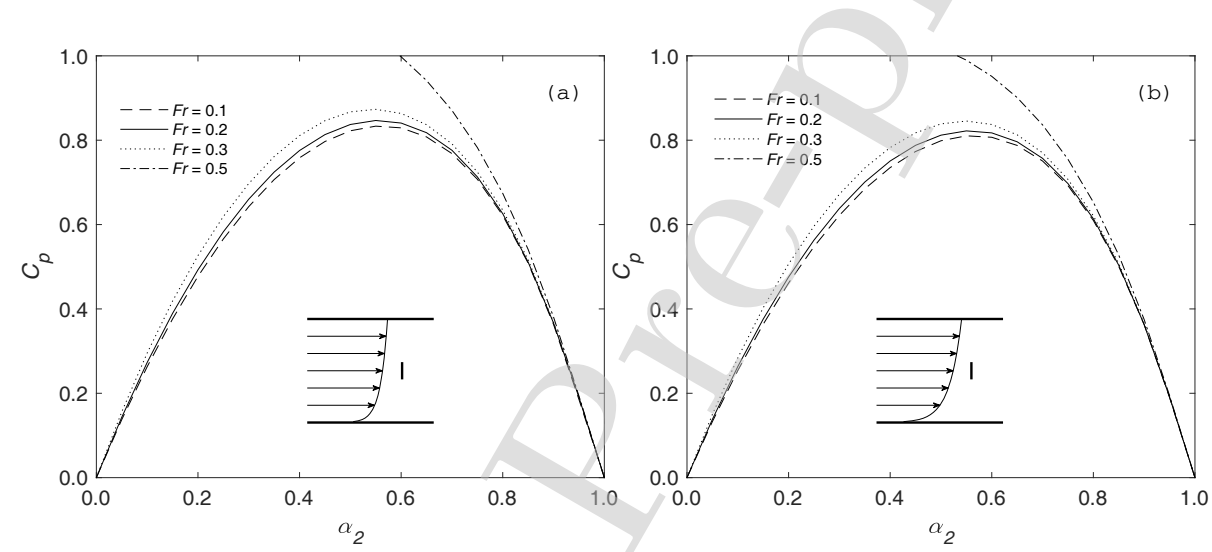

Figure 11: Power coefficient $C_{p}$ for a logarithmic velocity profile at Froude numbers $F r=0.1,0.2,0.3,0.5$, blockage ratio $B=1 / 6$, above: (a) sand bed; and (b) a gravel bed. The disc is deployed at mid-depth of the logarithmic shear flow
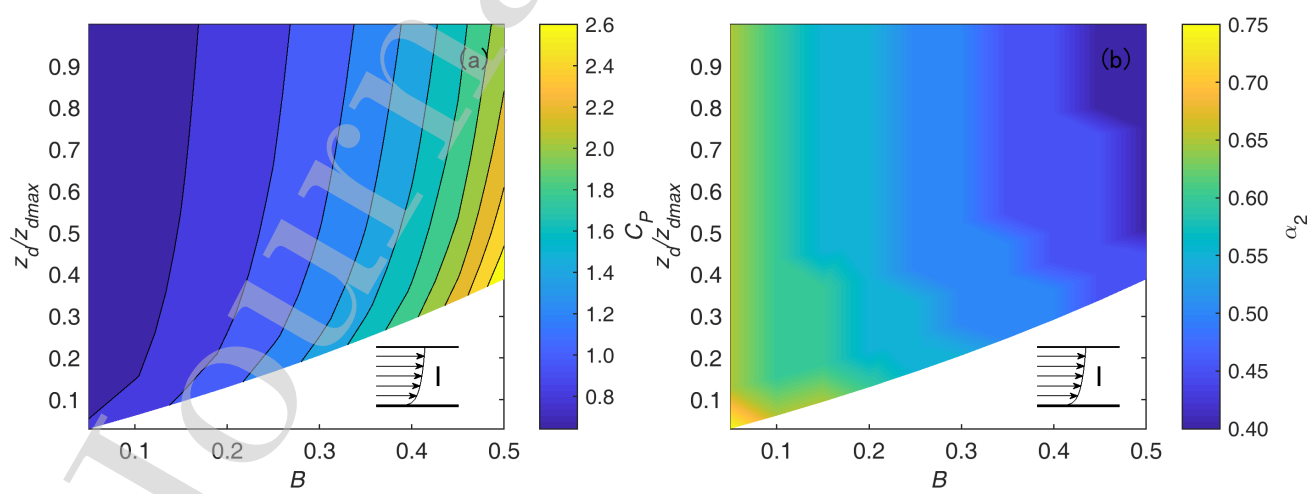

Figure 12: (a) Maximum power coefficient $C_{p}$ and (b) corresponding $\alpha_{2}$ for different blockage ratios $B$ and non-dimensional strip positions $z_{d} / z_{d \max }$, at Froude number $F r=0.1$ and logarithmic velocity profile $d_{50}=1 \mathrm{~mm}$. The disc is deployed at different elevations in the logarithmic shear flow for varing blockage ratio 
Figure 12 shows contours of maximum power coefficient $C_{p}$ and corresponding $\alpha_{2}$ with respect to blockage ratio $B$ and non-dimensional strip position $z_{d} / z_{d m a x}$, at $F r=0.1$ and for a logarithmic velocity profile corresponding to $d_{50}=1 \mathrm{~mm}$. Figure $12(\mathrm{a})$ indicates that a higher peak power coefficient is still achieved as the strip is moved downwards and has a higher blockage ratio, as in the asymmetric power law shear flow, and the efficiency exhibits greater dependency on blockage ratio.

\subsection{Basin efficiency for 2D cases}

We now take into account the basin efficiency for asymmetric power law velocity profiles of different shape $(n=0,1 / 5,1 / 7,1)$. Figure 13 shows that basin efficiency decreases monotonically as the thrust coefficient $C_{t}$ increases, for strips located at mid-depth. In general, an increase in shear (larger $n$ ) results in lower basin efficiency. However, an inverse trend can be observed when the strip is weak (large $\alpha_{2}$ or low $\left.C_{t}\right)$. This can be explained by considering the recovery process in the wake. As there is no momentum loss between Section 4 and Section 5, the energy loss between the two sections is determined by the uniformity of the velocity profiles at the two sections. For uniform flow cases, velocity recovery tends to increase the uniformity of the profile, leading to greater energy loss between the two sections. For non-uniform flow cases, however, changes in velocity may result in more non-uniform profiles and decreased energy loss. Such reduction in energy loss may further increase the basin efficiency. Figure 13 (a) shows the changes in basin efficiency $\eta$ as the power coefficient $C_{p}$ varies. All the lines $(n=0,1 / 7,1 / 5,1)$ in the figure have steep slopes near the maximum value of power coefficient, implying that the basin efficiency (environmental effect) can be reduced by decreasing the power coefficient. For example, in 1/7 power law shear flow, a $10 \%$ decrease in power coefficient can cause a $15 \%$ increase in basin efficiency.
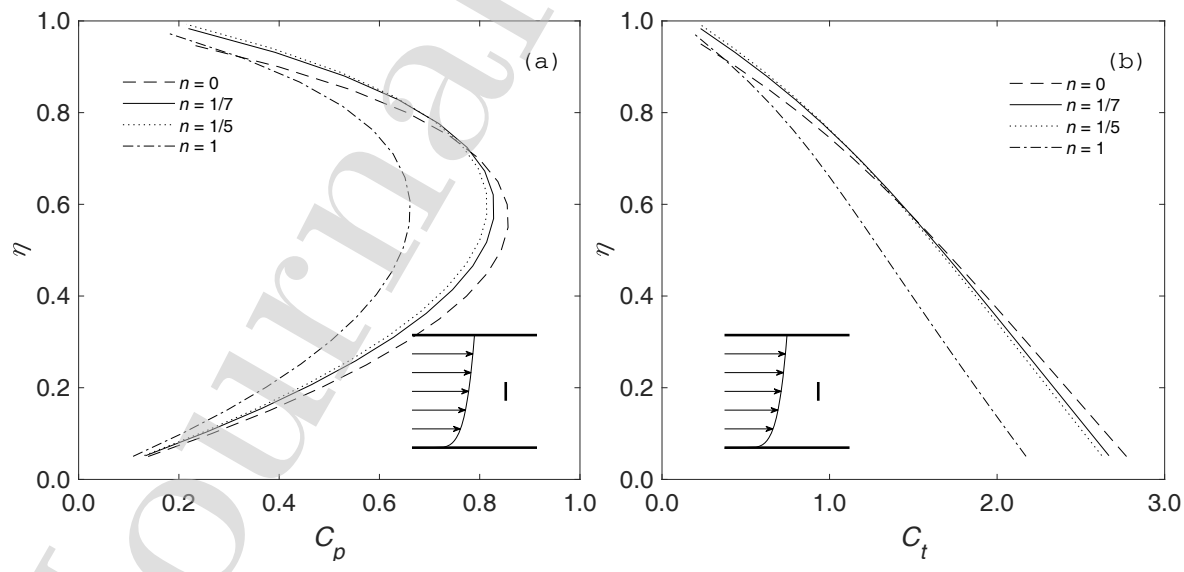

Figure 13: Basin efficiency $\eta$ as a function of (a) power coefficient $C_{p}$ and (b) thrust coefficient $C_{t}$ at Froude number $F r=0.1$, blockage ratio $B=1 / 6$, and asymmetric power law profiles of different shapes $(n=0,1 / 7,1 / 5,1)$, with strips located at mid-depth of the power-law shear flows

For logarithmic velocity profiles $\left(d_{50}=1 \mathrm{~mm}, 64 \mathrm{~mm}\right)$, similar relationships can be obtained between 
basin efficiency $\eta$, thrust coefficient $C_{t}$ and power coefficient $C_{p}$, as shown in Figures 14. Again for strips located at mid-depth, higher basin efficiency is achieved at smaller thrust coefficient $C_{t}$, and the basin efficiency is sensitive to the value of the power coefficient.
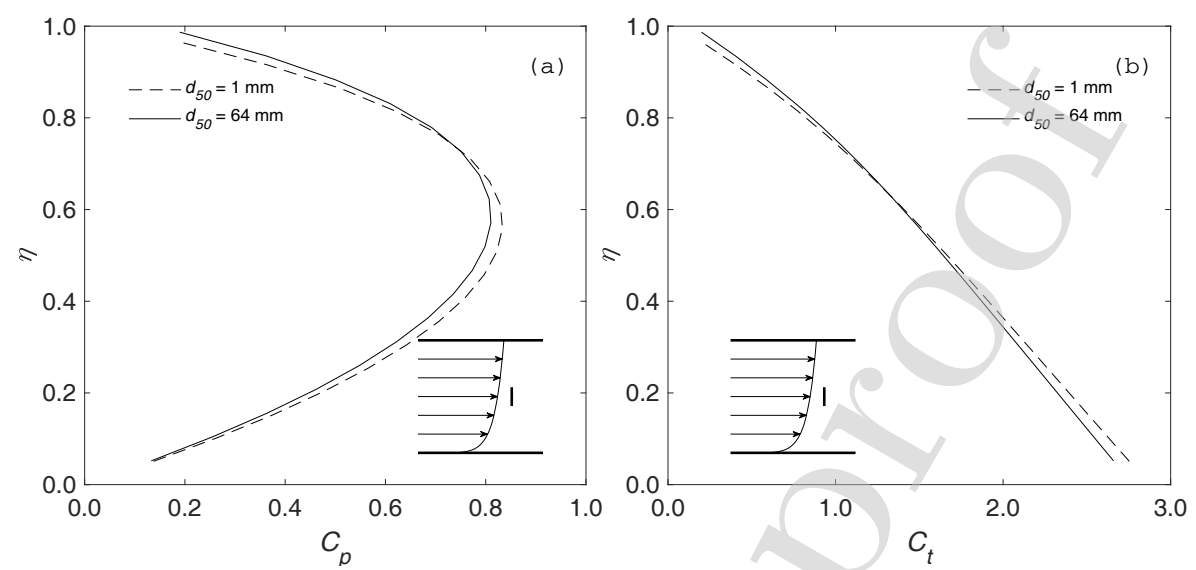

Figure 14: Basin efficiency $\eta$ as a function of (a) power coefficient $C_{p}$ and (b) thrust coefficient $C_{t}$ at Froude number $F r=0.1$, blockage ratio $B=1 / 6$, and logarithmic profiles of different shapes $\left(d_{50}=1 \mathrm{~mm}, 64 \mathrm{~mm}\right)$, with strips located at mid-depth of the logarithmic shear flow

\subsection{D Power law shear flow with vertical shear}

For arrays where the turbines are not very densely deployed, as in most designs to date, the inflow to each isolated turbines is likely to be a vertically-dominated shear flow, similar to the case of an isolated turbine operated in open water. For three-dimensional shear flow, the same procedure as for two dimensions can be applied. Consider a three-dimensional velocity profile with vertical shear described by a power law written as

$$
u_{1}\left(y, z_{b}\right)=U\left(\frac{z_{b}}{A_{1} / w_{1}}\right)^{n}
$$

where $y$ is the horizontal coordinate and is set to zero at the disc center. Elevation $z_{b}$ is zero at the bed and taken positive upwards. Thus the discharge in a micro flow tube confined by the two families of stream surfaces $\boldsymbol{\psi}=(\psi, \chi)$ is $d \psi\left(y, z_{b}\right) d \chi\left(y, z_{b}\right)=u_{1}\left(y, z_{b}\right) d y d z_{b}$. Taking the middle bottom point as zero reference, and integrating over a finite area gives

$$
\psi\left(y, z_{b}\right) \chi\left(y, z_{b}\right)=\int_{0}^{y} \int_{0}^{z_{b}} u_{1}\left(y, z_{b}\right) \mathrm{d} z_{b} \mathrm{~d} y=\frac{U A_{1} y}{w_{1}(n+1)}\left(\frac{z_{b}}{A_{1} / w_{1}}\right)^{n+1} .
$$

Without loss of generality, we set $\chi=y$, then $\psi$ and $\psi_{2}$ can be solved as

$$
\left\{\begin{array}{l}
\psi=\frac{U A_{1}}{w_{1}(n+1)}\left(\frac{z_{b}}{A_{1} / w_{1}}\right)^{n+1} \\
\chi=y
\end{array}\right.
$$


Representing $y$ and $z_{b}$ in terms of $\psi$ and $\chi$ using Equation 84 and substituting into Equation 82 gives

$$
u_{1}(\psi, \chi)=U^{1 /(n+1)}\left(\frac{w_{1}(n+1) \psi}{A_{1}}\right)^{n /(n+1)} .
$$

As with a $2 \mathrm{D}$ power law velocity profiles, the cross-sectional averaged velocity of a $3 \mathrm{D}$ vertical power law velocity profile is

$$
U=(n+1) \bar{U}
$$

Consider a round disc $\left(z_{b}-z_{d}\right)^{2}+y^{2}=R^{2}$. Then, the function for $\psi_{t}$ and $\chi_{t}$ used to represent the edges of the core incoming flow tube is given by

$$
\chi_{t}= \pm \sqrt{\alpha_{2} R^{2}-\left(\left(\frac{w_{1}(n+1)}{U A_{1}} \psi_{t}\right)^{1 /(n+1)} A_{1} / w_{1}-z_{d}\right)^{2}}
$$

460

where $\psi_{t} \in\left[\frac{U A_{1}}{w_{1}(n+1)}\left(\frac{z_{d}-D / 2}{A_{1} / w_{1}}\right)^{n+1}, \frac{U A_{1}}{w_{1}(n+1)}\left(\frac{z_{d}+D / 2}{A_{1} / w_{1}}\right)^{n+1}\right]$. Functions used to represent the edges of the overall control volume are

$$
\left\{\begin{array}{l}
\psi_{1}=0 \\
\psi_{2}=\frac{U A_{1}}{w_{1}(n+1)} \\
\chi_{1}=-\frac{w_{1}}{2} \\
\chi_{2}=\frac{w_{1}}{2}
\end{array}\right.
$$

where $\psi_{1}$ and $\psi_{2}$ denote the intersection of the $\psi$ stream surface family with Section 1 at the bed and water surface. $\chi_{1}$ and $\chi_{2}$ denote the intersection of the $\chi$ stream surface family with Section 1 at the left and right boundaries. Substitution of the above into Equations 29 to 30 enables calculation of $\alpha_{4}$ and $\beta_{4}^{\prime}$. The performance of the discs is evaluated using Equations 33.

Figure 15 shows the dependence of power coefficient $C_{p}$ on $\alpha_{2}$ given by Equation 33 for an actuator disc located at mid-depth in three-dimensional power law shear flows with vertical profiles of different shapes $(n=0,1 / 7,1 / 5)$. The water depth is set to 6 times the disc diameter. The Froude number defined using maximum velocity $U$ is $F r=0.1$. As with the two-dimensional cases, the figures show that increasing shear causes the power coefficient to decrease. However, there are always lateral gaps between neighbour discs in three-dimensional cases even when the actuator discs are densely deployed because of the round disc edges (the case in Figure 15(a), where the gap between neighbour discs at axis height is $S=0$ ); this results in a lower power coefficient compared to that of $2 D$ strips under the same depth conditions. For a given disc at a specific location, the submergence ratio (depth to disc diameter $D$ ) is determined. Then the larger the gap between discs, the lower the power coefficient. For the cases shown in Figure 15(b), variations in basin efficiency $\eta$ with thrust coefficient $C_{t}$ and power coefficient $C_{p}$ are given in Figure 16 . Trends in the $\eta$ lines are similar to those of their two-dimensional counterparts, with higher basin efficiency achieved at 
small thrust coefficient and either high or low values of power coefficient. Again, basin efficiency is sensitive
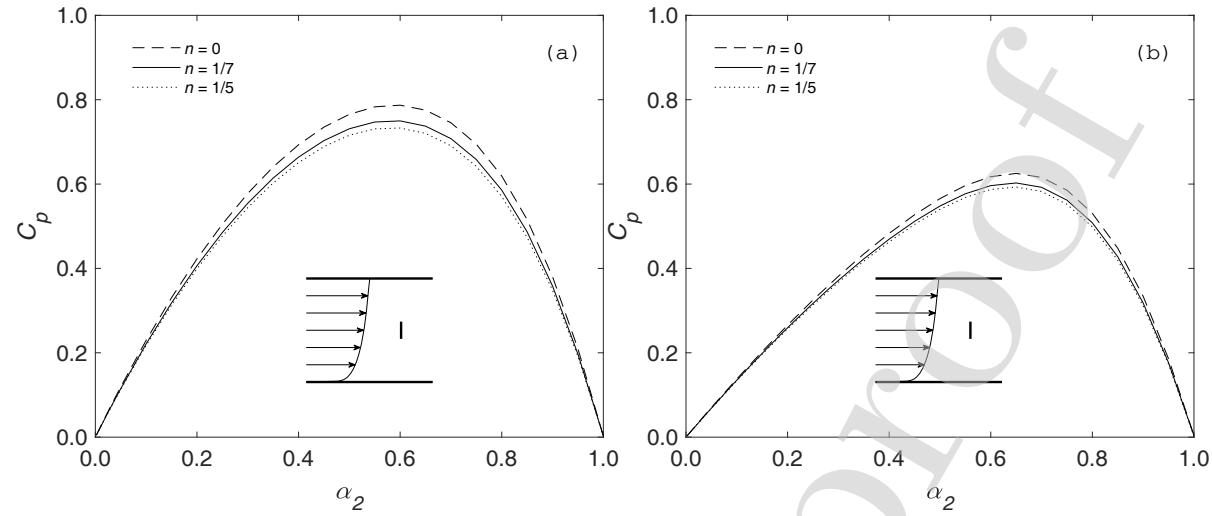

Figure 15: Variation in power coefficient $C_{p}$ with $\alpha_{2}$ for 3D power law velocity profiles $(n=0,1 / 7,1 / 5)$, at $F r=0.1$, Depth $=$ $6 D$ (rotor diameter) for: (a) $S=0$; and (b) $S=4 D$. The disc is deployed at mid-depth of the power-law shear flow and the rotor radius is $1 / 6$ of the water depth
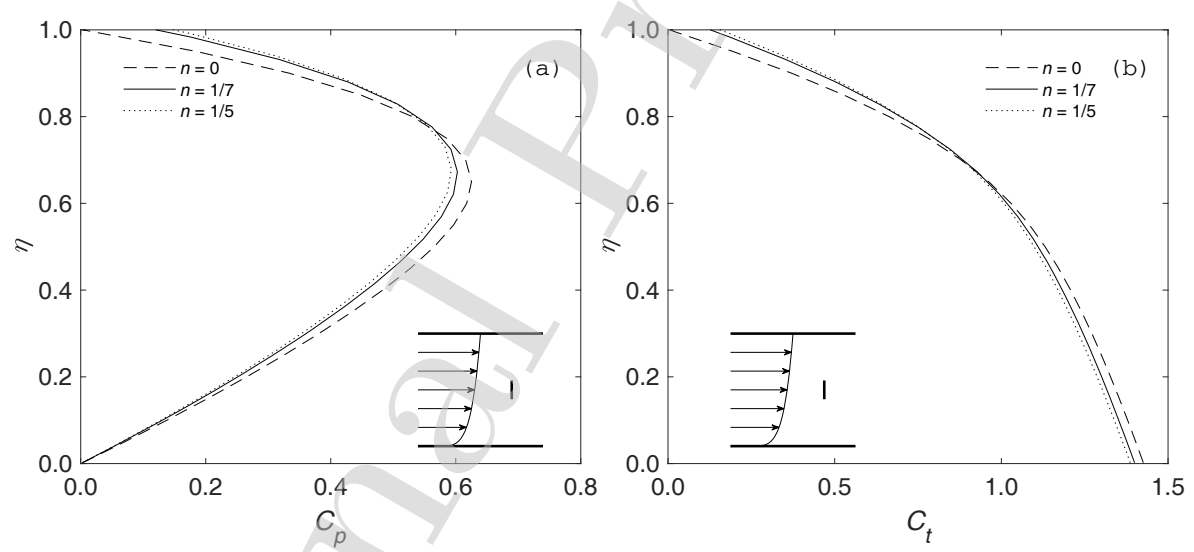

Figure 16: Basin efficiency $\eta$ as a function of (a) power coefficient $C_{p}$ and (b) thrust coefficient $C_{t}$ at $F r=0.1$, Depth $=6 D, S$ $=4 D$ for $3 \mathrm{D}$ power law velocity profiles $n=0,1 / 7,1 / 5$, the discs are located at mid-depth. The disc is deployed at mid-depth of the power-law shear flow and the rotor radius is $1 / 6$ of the water depth

To illustrate application of the model to realistic scenarios, case studies are conducted involving threedimensional shallow and deep channels. Figure 17 presents a schematic of the idealized turbine array layout where the turbines are evenly deployed in a single row. The inflow is uniform horizontally with a $1 / 7$ power law distribution in the vertical direction. Characteristics of the channels and turbines are shown in table 1, following Houlsby and Vogel (2016). The shallow channel has a depth of $30 \mathrm{~m}$, a width of $600 \mathrm{~m}$, and carries a flow with depth-averaged velocity $3 \mathrm{~m} / \mathrm{s}$, representing Strangford Lough. The deep channel has a 
(a)

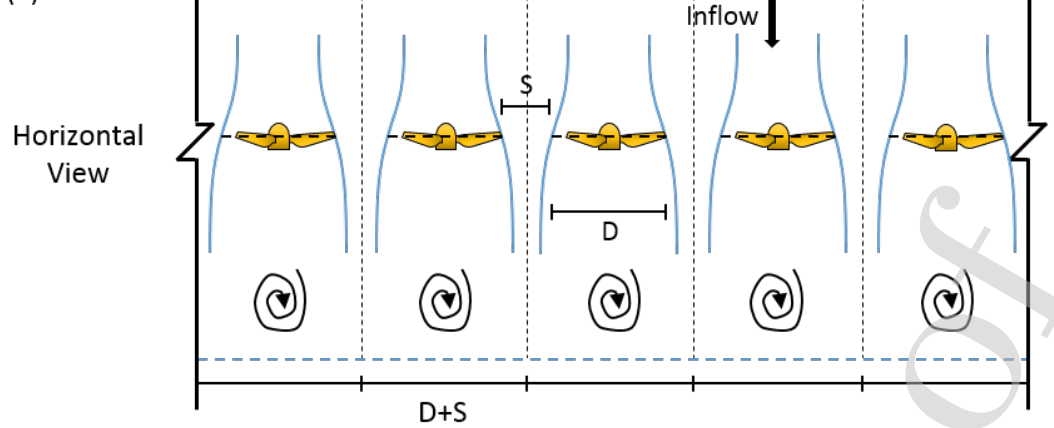

(b)

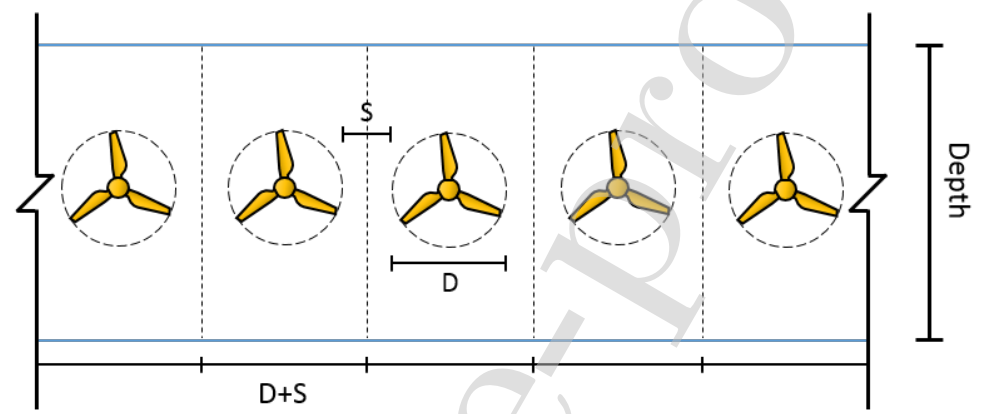

Figure 17: Turbine array layout: (a) plan view; (b) Vertical slice viewed from downstream. ( $D$ is turbine diameter, $S$ is gap between adjacent turbines)

depth of $50 \mathrm{~m}$, a width of $10 \mathrm{~km}$, and depth-averaged velocity of $2 \mathrm{~m} / \mathrm{s}$, representing the Pentland Firth. Each turbine diameter is $20 \mathrm{~m}$. As shown in table 1, vertical shear has slight effect on power, thrust, and basin efficiency for turbines located at mid-depth. However, blockage, power, thrust, and basin efficiency are sensitive to horizontal distance between neighbouring turbines.

Tidal turbines must be deployed at an elevation such that sufficient clearance is provided above the top rotor rims for submergence and below the bottom rotor rims for structural integrity. Thus for shallow channels, the mid-depth (as with the cases in table 1) provides a sensible approximation to the level at which the turbines are likely to be located. However for deep channels, there are more choices. For example, if the clearance distances are set to be $5 \mathrm{~m}$ both above and below the rotor in the deep channel cases, then the turbine axis may be located anywhere between $15 \mathrm{~m}$ to $35 \mathrm{~m}$ above the sea bed. We therefore take 15, 25 , and $35 \mathrm{~m}$ as representative axis heights for lower, medium, and upper turbine deployments respectively. Table 2 presents comparisons of turbine power, thrust, and basin efficiency, all of which increase as the turbine location moves from the lower part to the upper part of the water column. For the single turbine cases, a $60 \%$ increase in power and $15 \%$ increase in basin efficiency are achieved. Meanwhile, for the 500 turbine cases, the power and basin efficiency both increase by about $25 \%$. 


\begin{tabular}{|c|c|c|c|c|c|c|}
\hline \multirow{2}{*}{ Channel Type } & \multirow{2}{*}{ Feature } & \multicolumn{5}{|c|}{ Number of turbines } \\
\hline & & 1 & 10 & 30 & 100 & 500 \\
\hline \multirow{4}{*}{$\begin{array}{l}\text { Shallow Channel } \\
\text { (uniform) }\end{array}$} & Blockage & 0.017 & 0.17 & 0.52 & - & - \\
\hline & Power/turbine (MW) & 2.6 & 3.8 & 17 & - & - \\
\hline & Thrust/turbine (MN) & 1.3 & 2.3 & 19 & - & - \\
\hline & Basin efficiency & 0.65 & 0.55 & 0.28 & - & - \\
\hline \multirow{3}{*}{$\begin{array}{l}\text { Shallow Channel } \\
\text { (1/7 power law) }\end{array}$} & Power/turbine (MW) & 2.5 & 3.7 & 17 & - & - \\
\hline & Thrust/turbine (MN) & 1.3 & 2.2 & 19 & - & - \\
\hline & Basin efficiency & 0.62 & 0.57 & 0.29 & - & - \\
\hline \multirow{4}{*}{$\begin{array}{l}\text { Shallow Channel } \\
\text { (1/5 power law) }\end{array}$} & Power/turbine (MW) & 2.4 & 3.6 & 17 & - & - \\
\hline & Thrust/turbine (MN) & 1.3 & 2.3 & 18 & - & - \\
\hline & Basin efficiency & 0.62 & 0.52 & 0.30 & - & - \\
\hline & Blockage & 0.00070 & 0.0070 & 0.021 & 0.070 & 0.349 \\
\hline \multirow{3}{*}{$\begin{array}{c}\text { Deep Channel } \\
\text { (uniform) }\end{array}$} & Power/turbine (MW) & 0.74 & 0.75 & 0.78 & 0.86 & 1.8 \\
\hline & Thrust/turbine (MN) & 0.57 & 0.58 & 0.60 & 0.72 & 1.8 \\
\hline & Basin efficiency & 0.65 & 0.65 & 0.65 & 0.60 & 0.50 \\
\hline \multirow{3}{*}{$\begin{array}{l}\text { Deep Channel } \\
\text { (1/7 power law) }\end{array}$} & Power/turbine (MW) & 0.76 & 0.77 & 0.79 & 0.88 & 1.8 \\
\hline & Thrust/turbine (MN) & 0.56 & 0.57 & 0.59 & 0.71 & 1.8 \\
\hline & Basin efficiency & 0.67 & 0.67 & 0.67 & 0.62 & 0.52 \\
\hline \multirow{3}{*}{$\begin{array}{l}\text { Deep Channel } \\
\text { (1/5 power law) }\end{array}$} & Power/turbine (MW) & 0.75 & 0.76 & 0.78 & 0.87 & 1.8 \\
\hline & Thrust/turbine (MN) & 0.55 & 0.56 & 0.58 & 0.70 & 1.7 \\
\hline & Basin efficiency & 0.68 & 0.68 & 0.68 & 0.63 & 0.52 \\
\hline
\end{tabular}

Table 1: Power, thrust, and basin efficiencies for different numbers of turbines located in three-dimensional shallow and deep channels 


\begin{tabular}{ccccccc}
\hline \multirow{2}{*}{ Channel Type } & Feature & \multicolumn{5}{c}{ Number of turbines } \\
\cline { 3 - 7 } & Blockage & 1 & 10 & 30 & 100 & 500 \\
\hline \multirow{2}{*}{ Deep Channel } & 0.00070 & 0.0070 & 0.021 & 0.070 & 0.349 \\
(lower) & Power/turbine (MW) & 0.56 & 0.57 & 0.59 & 0.68 & 1.6 \\
& Thrust/turbine (MN) & 0.45 & 0.46 & 0.52 & 0.59 & 1.8 \\
Deep Channel & Basin efficiency & 0.62 & 0.62 & 0.58 & 0.57 & 0.43 \\
(medium) & Power/turbine (MW) & 0.76 & 0.77 & 0.79 & 0.88 & 1.8 \\
& Thrust/turbine (MN) & 0.56 & 0.57 & 0.59 & 0.71 & 1.8 \\
Deep Channel & Basin efficiency & 0.67 & 0.67 & 0.67 & 0.62 & 0.52 \\
(upper) & Power/turbine (MW) & 0.90 & 0.91 & 0.93 & 1.0 & 2.0 \\
& Thrust/turbine (MN) & 0.64 & 0.64 & 0.66 & 0.79 & 1.8 \\
& Basin efficiency & 0.71 & 0.71 & 0.71 & 0.65 & 0.54 \\
\hline
\end{tabular}

Table 2: Power, thrust, and basin efficiencies of different numbers of turbines located at lower, middle, and upper elevations in the water column of a three-dimensional deep channel

\section{Discussion and conclusion}

An analytical model based on LMADT has been proposed to estimate the power extracted by idealized turbines in shear flow, allowing the combined effect of vertical shear and gravity on idealized turbine performance to be investigated. The model is first established in three spatial dimensions under the assumption that pressure recovers much faster than velocity. Then simplification is made by adopting a second assumption of self-similar wake profiles. This assumption is applicable to actuator discs with uniform local resistance, which is the case when the geometric blockage and shear are not excessive. Finally, the basin efficiency is determined by assuming the velocity profile far downstream of the turbines is similar to that of the upstream flow.

First, the model demonstrations are conducted based on two- and three-dimensional cases with shear 15 idealized turbine performance, where the profiles of upstream flow are described by power and logarithmic laws. Analytical solutions indicate that: (i) for free surface flows, the gravity effect improves the performance of actuator discs by creating a water level drop between the upstream and downstream ends of the strip, which in turn provides an extra source of energy; (ii) the shear effect can either improve or diminish disc performance by inducing upstream and bypass flow velocities that are different to each other. Actuator discs located at high velocity regions have lower power coefficients, whereas turbines located at low velocity regions have higher power coefficients; (iii) basin efficiency is sensitive to power coefficient, implying that 

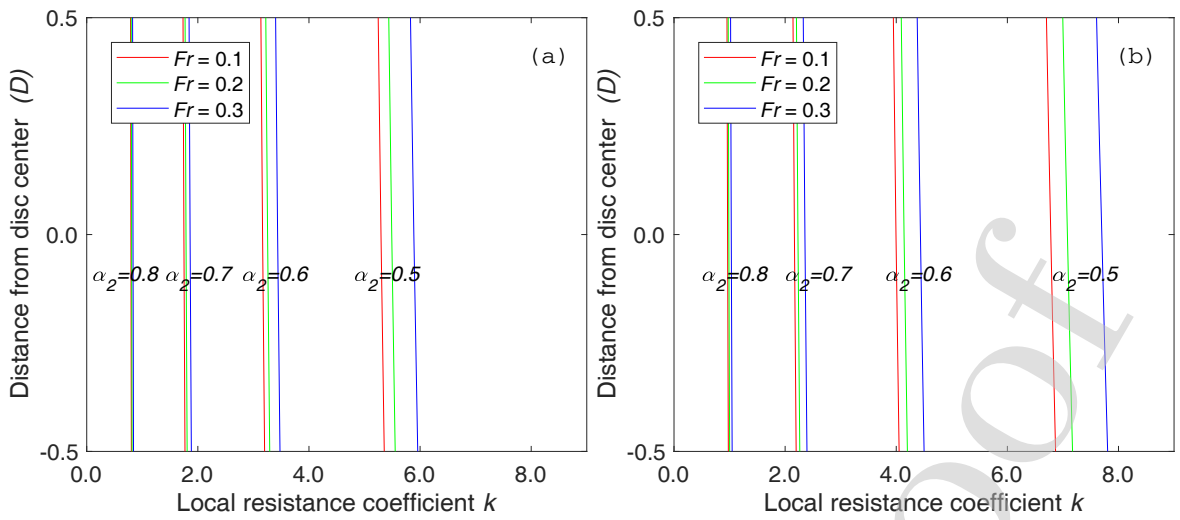

Figure 18: Profiles of local resistance coefficient $k$ along the discs for asymmetric power law velocity profile with $n=1 / 7$, at blockage ratio $B=1 / 6$ and Froude numbers $F r=0.1,0.2,0.3$, the discs are deployed at (a) mid-depth; and (b) bottom

environmental impact can be significantly reduced by decreasing the power coefficient. Within the range of the present study, an approximately $15 \%$ increase in basin efficiency can be achieved by setting the actuator discs to operate at $90 \%$ of the peak power coefficient; and (iv) within the range of the three-dimensional cases considered, reduction in horizontal spacing between adjacent idealized turbines suppresses vertical shear for turbines located at mid-depth. However, for deep channels with specific numbers of turbines, vertical shear is important regarding power extracted, structure load, and basin efficiency. The increase in power can reach $60 \%$ if the turbine location is altered from the lower to the upper part of the water column, and the associated increase in basin efficiency can be as high as $25 \%$.

As mentioned in $\S 2$, specific resistance distributions (Equation 41 - Equation 43) are used to achieve self-similar core wake profiles, appropriate for the cases studied. To provide more details of the disc properties, the local resistance coefficient $k$ for the asymmetric power law cases in Figure 10 are calculated, and shown in Figure 18 (a). It can be seen that $k$ is almost uniform along the disc, although slightly inclined. When $a_{2}$ is close to 1 (i.e. weak disc), the resistance profiles for discs in flows at different Froude numbers (indicated using different colours) overlap, whereas for smaller $a_{2}$ values the lines separate out from each other. Flows at larger Froude number require discs with larger resistance. As the discs are moved from mid-depth to the bottom, the resistance $k$ increases without too much change to profile shape. Hence, for $1 / 7$ power law shear flows and discs with mild blockages, the assumption of self-similar core wake profiles is compatible with (commonly utilised) uniform resistance discs.

In reality, some energy is continuously dissipated as heat even in the absence of discs. This phenomenon is associated with: (1) the water head difference between channel ends which drives flow acceleration; and (2) 

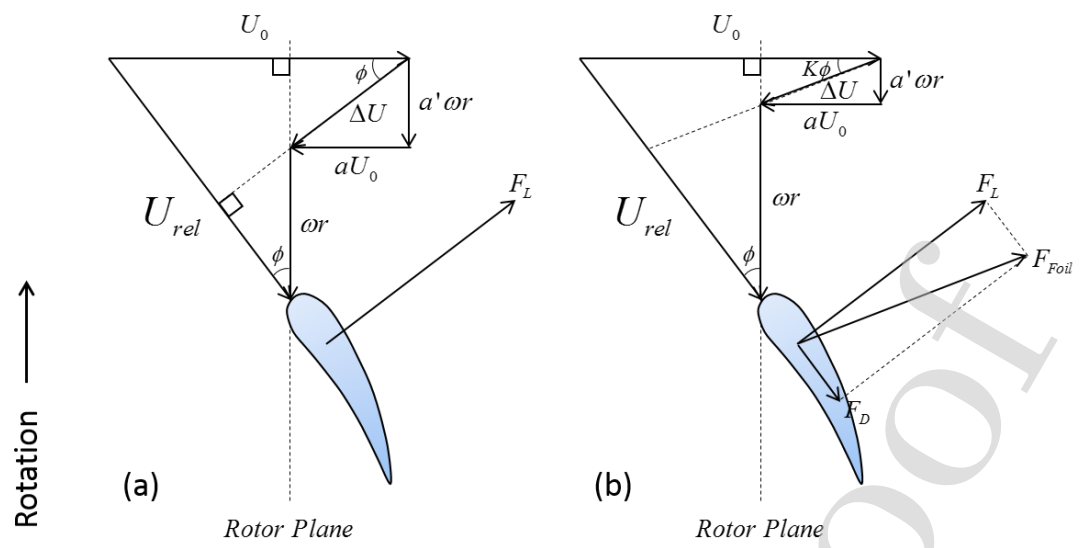

Figure 19: Schematic of forces applied on and velocity variations at (a) an ideal foil section; and (b) an actual foil section

bed resistance causing flow deceleration. To maintain balance between these two effects, some mechanical energy of the water is consumed through flow shear. The inclusion of artificial energy extraction alters the natural dissipation process, adding to the total dissipation due to wake mixing. By ignoring energy loss due to natural processes, the basin efficiency herein is simply a first-order approximation.

The most significant difference between the scenarios of an actuator disc (i.e. idealized turbine) and an actual turbine arises from the rotation of the blades. It is possible to compare the two scenarios using classical actuator disc theory and rotating blade momentum theory. From a foil-scale perspective, the foil has to move in a circular orbit in order to extract energy from the fluid. Two key points should be noted concerning this process. Firstly, rotation of the blades causes the wake to rotate. When the flow passes through the rotor, the energy flux within a steam tube is diverted and partially dissipated before it returns to the $x$ direction. This phenomenon can be viewed as an additional energy loss from the water but is treated as part of the energy extracted by the disc in the actuator disc framework. Secondly, an ideal foil does not exist and so the foil drag coefficient is never zero in moving water. This induces a spurious drag force $F_{D}$ (as shown in Figure $\left.19(\mathrm{~b})\right)$ ) and causes additional energy loss from the water in a similar way to a submerged structure; this is inherently considered as part of energy extracted by the disc in the actuator disc framework. To sum up, only when the turbine blades rotate rapidly and the drag coefficient for blade sections is small, do actuator disc and rotors become equivalent.

We examine the difference between actuator disc and a rotor by depicting the power coefficient ratio (power coefficient normalized by Betz limit) for a rotor with non-ideal foil sections (Figure 20), where $K$ indicates the importance of lift $F_{L}$ with respect to the resultant force $F_{F o i l}$. When the foil drag $F_{D}$ us not equal to zero, the resultant force $F_{F o i l}$ is no longer aligned with $F_{L}$ and so is not perpendicular to the 
relative incident velocity $U_{\text {rel }}$ (as shown in Figure 19). In this case, the angle $(K \phi)$ between the velocity variation $\Delta U$ and incident velocity $U_{0}$ is smaller than that $(\phi)$ between the relative incident velocity $U_{r e l}$

and rotor plane. $K$ is defined as the ratio between the two angles. As expected, when $K=1$ (i.e. foil drag can be ignored), the optimum power coefficient approaches the Betz limit as the tip speed ratio $\lambda$ increases. Meanwhile for cases where $K<1$, (note: foil drag becomes more important as $K$ decreases), the optimum power coefficient approaches a limit lower than the Betz limit. For the majority of actual turbines, $\lambda$ is likely to be larger than 4 , which corresponds to the mild-sloped portions of the curves. This implies that foil drag is mainly responsible for the difference between optimum power coefficient of an actuator disc and an actual rotor. From the foregoing discussion, we observe that actuator disc theory regards energy loss from the rotating wake and foil drag as contributing to energy extracted by the disc. This means actuator disc theory may overestimate energy coefficients for turbines and so provides an upper limit for energy assessment.

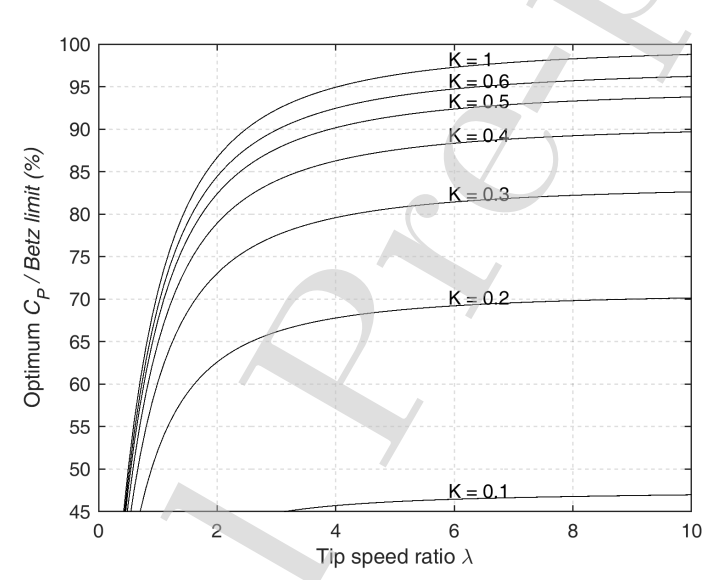

Figure 20: Comparison between power coefficient of an actuator disc (idealized turbine) and a real turbine ( $K$ indicates the relative importance of lift $F_{L}$ to resultant force $\left.F_{F o i l}\right)$

The proposed model provides an efficient method to investigate submerged structures in free surface shear flows. Beside considering the performance of single row water turbines, the model can also be used to study the loads acting on offshore structures.

\section{Acknowledgement}

The authors would like to acknowledge support from the National Natural Science Foundation of China (20161300680, grant number: 51679125), and useful discussions with Dr Ton van den Bremer of the University of Oxford. The first author would also like to acknowledge financial support from the China Scholarship Council (No. 201606210444). 


\section{References}

\section{References}

Adcock T, Draper S, Houlsby G, Borthwick A, Serhadlığlu S. The available power from tidal stream turbines in the Pentland Firth. Proceedings of the Royal Society of London A: Mathematical, Physical and Engineering Sciences 2013;469(2157). doi:10.1098/rspa.2013.0072.

Bahaj A, Molland A, Chaplin J, Batten W. Power and thrust measurements of marine current turbines under various hydrodynamic flow conditions in a cavitation tunnel and a towing tank. Renewable energy 2007;32(3):407-26.

Bahaj A, Myers L, Rawlinson-Smith R, Thomson M. The effect of boundary proximity upon the wake structure of horizontal axis marine current turbines. Journal of Offshore Mechanics and Arctic Engineering 2012;134(2):021104.

Barnsley M, Wellicome J. Final report on the 2nd phase of development and testing of a horizontal axis wind turbine test rig for the investigation of stall regulation aerodynamics. Carried out under ETSU Agreement E A 1990;5.

Betz A. Das maximum der theoretisch möglichen ausnützung des windes durch windmotoren. Zeitschrift für das gesamte Turbinenwesen 1920;26(307-309):8.

Draper S. Tidal stream energy extraction in coastal basins. Univ Oxford, DPhil Thesis $2011 ;: 253$.

Draper S, Nishino T. Centred and staggered arrangements of tidal turbines. Journal of Fluid Mechanics 2014;739:72-93.

Draper S, Nishino T, Adcock T, Taylor P. Performance of an ideal turbine in an inviscid shear flow. Journal of Fluid Mechanics 2016;796:86-112.

Fallon D, Hartnett M, Olbert A, Nash S. The effects of array configuration on the hydro-environmental impacts of tidal turbines. Renewable Energy 2014;64:10-25.

Froude R. On the part played in propulsion by differences of fluid pressure. Transactions of the Institute of Naval Architects 1889;30(390-405):22.

Garrett C, Cummins P. The power potential of tidal currents in channels. Proceedings of the Royal Society of London A: Mathematical, Physical and Engineering Sciences 2005;461(2060):2563-72.

Garrett C, Cummins P. The efficiency of a turbine in a tidal channel. Journal of Fluid Mechanics 2007;588:243-51.

Gauthier E, Kinsey T, Dumas G. Impact of blockage on the hydrodynamic performance of oscillating-foils hydrokinetic turbines. Journal of Fluids Engineering 2016;138(9):091103.

González-Gorbeña E, Rosman P, Qassim R. Assessment of the tidal current energy resource in São Marcos Bay, Brazil. Journal of Ocean Engineering and Marine Energy 2015;1(4):421-33.

Houlsby G, Draper S, Oldfield M. Application of linear momentum actuator disc theory to open channel flow. Report no OUEL 2008;2296(08).

Houlsby G, Vogel C. The power available to tidal turbines in an open channel flow. Proceedings of the Institution of Civil Engineers-Energy $2016 ;: 1-10$.

Hunter W, Nishino T, Willden R. Investigation of tidal turbine array tuning using 3D Reynolds-Averaged Navier-Stokes simulations. International Journal of Marine Energy 2015;10:39-51.

Joukowsky N. Windmill of the NEJ type. Transactions of the Central Institute for Aero-hydrodynamics of Moscow 1920;1:57.

Karsten R, Swan A, Culina J. Assessment of arrays of in-stream tidal turbines in the Bay of Fundy. Phil Trans R Soc A 2013;371(1985):20120189.

Kinsey T, Dumas G. Impact of channel blockage on the performance of axial and cross-flow hydrokinetic turbines. Renewable Energy 2017;103:239-54.

Lanchester F. Contribution to the theory of propulsion and the scew propeller. Transaction of the Institution of Naval Architects, Vol LVII, March 1915;25:98-116.

Masatsuka K. I do Like CFD, vol. 1. volume 1. Lulu. com, 2013. 
Nash S, O'Brien N, Olbert A, Hartnett M. Modelling the far field hydro-environmental impacts of tidal farms-a focus on tidal regime, inter-tidal zones and flushing. Computers \& Geosciences 2014;71:20-7.

Nash S, Olbert A, Hartnett M. Towards a low-cost modelling system for optimising the layout of tidal turbine arrays. Energies 2015;8(12):13521-39.

Nishino T, Willden R. The efficiency of an array of tidal turbines partially blocking a wide channel. Journal of Fluid Mechanics 2012;708:596-606.

Nishino T, Willden R. Two-scale dynamics of flow past a partial cross-stream array of tidal turbines. Journal of Fluid Mechanics 2013;730:220-44.

Okulov V, van Kuik G. The Betz-Joukowsky limit: on the contribution to rotor aerodynamics by the British, German and Russian scientific schools. Wind Energy 2012;15(2):335-44.

Rankine W. On the mechanical principles of the action of propellers. Transactions of the Institute of Naval Architects $1865 ; 13: 13-30$.

Santo H, Taylor P, Williamson C, Choo Y. Current blockage experiments: force time histories on obstacle arrays in combined steady and oscillatory motion. Journal of Fluid Mechanics 2014;739:143-78.

Schlichting H. Boundary layer theory. McGraw-Hill, 1955.

Sørensen J. Aerodynamic aspects of wind energy conversion. Annual Review of Fluid Mechanics 2011;43:427-48.

Soulsby R. Dynamics of marine sands: a manual for practical applications. Thomas Telford, 1997.

Taylor P. Current blockage: reduced forces on offshore space-frame structures. In: Offshore Technology Conference. Offshore Technology Conference; 1991. .

Taylor P, Santo H, Choo Y. Current blockage: Reduced Morison forces on space frame structures with high hydrodynamic area, and in regular waves and current. Ocean Engineering 2013;57:11-24.

Vennell R. Tuning turbines in a tidal channel. Journal of Fluid Mechanics 2010;663:253-67.

Vennell R. Tuning tidal turbines in-concert to maximise farm efficiency. Journal of Fluid Mechanics 2011;671:587-604.

Wentworth C. A scale of grade and class terms for clastic sediments. The Journal of Geology 1922;30(5):377-92.

Whelan J, Graham J, Peiro J. A free-surface and blockage correction for tidal turbines. Journal of Fluid Mechanics 2009;624:28191.

Whelan J, Thomson M, Graham J, Peiro J. Modelling of free surface proximity and wave induced velocities around a horizontal axis tidal stream turbine. In: Proceedings of the 7th European Wave and Tidal Energy Conference. 2007. .

Wimshurst A, Willden R. Tidal power extraction on a streamwise bed slope. Ocean Engineering 2016;125:70-81. 